\title{
Synergistic Value in Vertically Integrated Power-to-Gas Energy Systems
}

\author{
Gunther Glenk* \\ Business School, University of Mannheim, Mannheim 68131, Germany \\ TUM School of Management, Technical University of Munich, Munich 80333, Germany, glenk@uni-mannheim.de \\ Stefan Reichelstein* \\ Business School, University of Mannheim, Mannheim 68131, Germany \\ Graduate School of Business, Stanford University, Stanford, California 94305, USA, reichelstein@stanford.edu
}

\begin{abstract}
$I^{n}$ $\mathrm{n}$ vertically integrated energy systems, integration frequently entails operational gains that must be traded off against the requisite cost of capacity investments. In the context of the model analyzed in this study, the operational gains are subject to inherent volatility in both the price and the output of the intermediate product transferred within the vertically integrated structure. Our model framework provides necessary and sufficient conditions for the value (NPV) of an integrated system to exceed the sum of two optimized subsystems on their own. We then calibrate the model in Germany and Texas for systems that combine wind energy with Power-to-Gas (PtG) facilities that produce hydrogen. Depending on the prices for hydrogen in different market segments, we find that a synergistic investment value emerges in some settings. In the context of Texas, for instance, neither electricity generation from wind power nor hydrogen production from PtG is profitable on its own in the current market environment. Yet, provided both subsystems are sized optimally in relative terms, the attendant operational gains from vertical integration more than compensate for the stand-alone losses of the two subsystems.
\end{abstract}

Key words: operational volatility; vertical integration; capacity investment; renewable energy; power-to-gas History: Received: July 2019; Accepted: October 2019 by Kalyan Singhal after one revision.

\section{Introduction}

As the global efforts to reduce carbon dioxide emissions associated with burning fossil fuels gain urgency, there is a growing sense that a range of industrial processes will need to be decarbonized in addition to power generation. The production of industrial gases provides a case in point. The predominant method of producing hydrogen, for instance, is through steam methane reforming which relies on natural gas as its energy feedstock and results in significant carbon dioxide emissions (Davis et al. 2018). A decarbonized alternative to steam methane reforming is to obtain hydrogen via a Power-to-Gas (PtG) process based on water electrolysis whereby electricity infused in water instantly splits the water molecule into oxygen and hydrogen. ${ }^{1}$

The widely reported rapid decline in the cost of renewable electricity through wind or solar photovoltaic installations naturally raises the question as to

This is an open access article under the terms of the Creative Commons Attribution License, which permits use, distribution and reproduction in any medium, provided the original work is properly cited. whether a PtG system combined with an upstream renewable power source is becoming cost competitive with traditional, carbon-intensive ways of producing hydrogen. A PtG system that is vertically integrated with a renewable power source will also have the financial advantage of avoiding the transaction cost typically reflected in the mark-up of electricity prices for buying rather than selling electricity. ${ }^{2}$

The intermittency of power generated from wind or solar photovoltaic sources presents a well-known problem in balancing electricity supply and demand in real-time. ${ }^{3}$ One potential remedy suggested is to divert surplus energy from renewable power sources to the production of energy storing products like hydrogen. At the same time, though, the inherent intermittency of renewable power generation negatively affects the economics of a vertically integrated $\mathrm{PtG}$ system due to the high opportunity cost of "starving" the expensive PtG system at times of low output from the renewable power source; see Glenk and Reichelstein (2019). That bottleneck can be alleviated by allowing the PtG system to also draw power from the grid, though the resulting hydrogen will then become more expensive on account of facing the buying rather than the selling price of electricity. 
Furthermore, the hydrogen produced will be decarbonized only to the extent that grid power is decarbonized.

The front-part of this study analyzes a generic model of a vertically integrated production system. The downstream unit requires an intermediate production input (e.g., power) that can be sourced from the external market, or alternatively from an upstream unit. The output of the upstream unit is inherently volatile as it fluctuates in an exogenous fashion across the hours of the year. Depending on the current market price of the intermediate input, the vertically integrated system can decide on a real-time basis to what extent the intermediate input is sold on the external market or transferred to the downstream unit. In contrast to some of the recent work on production synergies, we capture volatility not by random shocks, but by predictable variations in both the level of the intermediate input produced and its market price (Hekimoglu et al. 2017, Kouvelis et al. 2018, de Véricourt and Gromb 2018).

The main question posed in the modeling part is whether a vertically integrated energy system exhibits synergistic investment value. Our criterion for such synergies is that the net present value (NPV) of the vertically integrated system exceeds the sum of the optimized NPVs of the two stand-alone facilities that would either sell or buy the intermediate input exclusively on the external market. In this comparison, zero will always be a lower bound for the optimized NPV of the two stand-alone entities because of the option not to invest in capacity in the first place. If either or both energy systems have negative NPVs on their own, the presence of a synergistic investment value must entail operational gains which more than compensate for the cost of capacity investments that would be uneconomical on their own in case the two subsystems were to operate in stand-alone mode. ${ }^{4}$ The conditions for a synergistic investment value are straightforward to identify in a hypothetical stationary environment where electricity prices and output do not vary over time. Our analysis demonstrates how these conditions extend to production environments that are subject to operational volatility.

Our model analysis shows that the emergence of a synergistic investment value hinges critically on the two subsystems being sized optimally in terms of their relative capacity. ${ }^{5}$ The need for this optimization reflects that in settings where the value of capacity investments is large relative to the annual operating costs, overall profitability is highly sensitive to trading off volatile revenue opportunities against idle capacity. We demonstrate that the optimal relative capacity size of two subsystems can be expressed in terms of a few aggregate variables. These comprise the life-cycle cost of the intermediate and the final product, and the time-averaged price and output levels, with the latter two averages adjusted by covariance terms that reflect the extent to which intertemporal variations in prices correlate with variations in output from the upstream unit.

The back-end of our analysis calibrates our model in the context of PtG hydrogen production facilities that are co-located with wind parks. We provide a numerical evaluation for vertically integrated energy systems in both Germany and Texas, two jurisdictions that have installed substantial amounts of wind power in recent years. On a stand-alone basis, wind parks are currently unprofitable in Texas, though they entail positive NPVs in Germany, in large part due to public subsidies for renewable energy. The stand-alone value of investments in PtG facilities depends on the attainable market price of hydrogen. For medium-scale supply settings, hydrogen sales prices tend to be relatively high, making stand-alone PtG facilities marginally profitable in both Germany and Texas. In contrast, such facilities entail negative NPVs in both jurisdictions relative to the lower prices associated with industrial-scale hydrogen supply arrangements.

Since the integrated system generally experiences some operational gains from the avoided transaction costs that arise in the intermediate input market, one would expect a synergistic investment value to emerge if both wind power and hydrogen production are cost competitive (profitable) on their own. We confirm this for the setting of Germany and mediumscale hydrogen supply. Conversely, it may intuitively appear difficult for the synergistic effect to be sufficiently large so as to outweigh stand-alone losses if those occur in both subsystems. Nonetheless, we do identify such a synergistic investment value in the context of Texas where neither wind power nor hydrogen production is economically viable by itself.

An instructive metric for quantifying the gains from vertical integration is what we term the break-even price of hydrogen for a vertically integrated energy system. The break-even price is defined as the lowest downstream (i.e., hydrogen) price at which the vertically integrated system achieves a synergistic investment value. By construction, the break-even hydrogen price of the vertically integrated system is always lower than the price at which hydrogen production turns profitable on its own. In the context of Texas and industrial-scale hydrogen supply, we find that the break-even price of hydrogen for a vertically integrated energy system is about 30\% lower than the critical price at which hydrogen would become viable on its own. This difference illustrates the relative magnitude of the synergistic gains in that particular market context. 
We finally project likely improvements in the economics of combined energy systems that integrate wind power with hydrogen production. Several factors are likely to contribute to more robust synergistic investment values in the future. These include sustained price reductions for both wind turbines and PtG facilities as well as greater operational volatility in terms of fluctuating market prices for electricity. The latter trend is mainly a consequence of the trend towards time-of-use pricing. Overall, our projections indicate that even relative to the benchmark of the low hydrogen prices associated with large-scale industrial supply, synergistic investment value for the integrated systems will widely emerge in both Texas and Germany within a decade. These projections take into account that the public support for wind energy, e.g., the production tax credit (PTC) available in the United States is scheduled to be phased out in the coming years.

For the specific application of wind power combined with hydrogen production, our numerical assessments point to more favorable economics than other recent studies (Ainscough et al. 2014, Bertuccioli et al. 2014, Felgenhauer and Hamacher 2015, Glenk and Reichelstein 2019). We attribute this difference to the fact that our calculations are based on subsystems that have been sized optimally, an aspect that is of first-order importance when capacity investments account for a large share of overall production costs. In addition, our calculations take advantage of higher capacity utilization that results when both renewable and grid electricity are converted to hydrogen. Finally, our calculations reflect more recent cost and operational inputs for wind energy and PtG.

The remainder of the study is organized as follows. Section 2 develops the model framework for the identification of synergistic investment value in vertically integrated energy systems under conditions of operational volatility. Section 3 applies the model framework to PtG and wind energy. We first provide an assessment based on most recent data and then project likely changes in synergistic investment values for the coming decade. Section 5 concludes the paper. Supplemental materials such as proofs and data sources are provided in the Appendix.

\section{Model Framework}

Our model framework considers a vertically integrated energy system that comprises two interacting subsystems. For concreteness, we focus on a renewable energy source, like wind or solar power that is connected with a PtG facility. In our applications, the gas will be hydrogen that is produced via an electrolysis process. The setting in Figure 1 comprises four building blocks: the renewable energy source, the PtG
Figure 1 Illustration of the Vertically Integrated Energy System [Color figure can be viewed at wileyonlinelibrary.com]

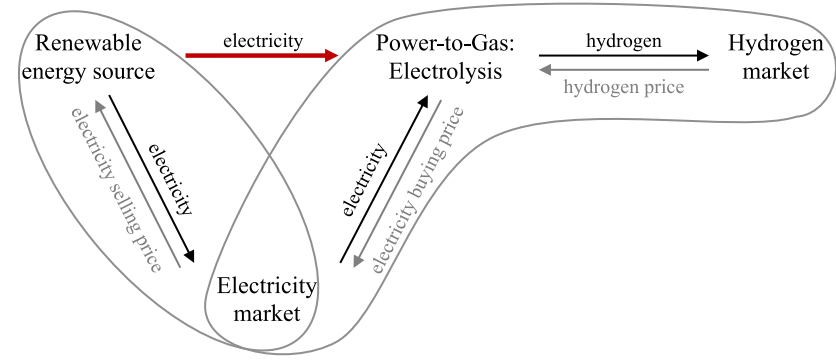

facility, and external markets for both electricity and hydrogen.

We note that some ingredients of our model have been captured in earlier related work. For instance, in Hu et al. (2015), the PtG facility in our context is effectively replaced by an electricity customer who can obtain power from either the grid or his own renewable energy source. Alternatively, Kazaz (2004) studies an upstream unit that is an olive plantation facing fluctuating output levels and prices for olives, while the downstream unit is an olive press that converts olives into oil sold at a stable price. Similarly, it could be advantageous to combine an upstream pump for crude oil, that is subject to fluctuations in output and prices over time, with a downstream refinery system (Dong et al. 2014).

As a stand-alone operating unit, the renewable energy source in Figure 1 can generate electricity that is sold on the open market at time-varying prices. This stand-alone subsystem is represented through the ellipse on the left. The boomerangshaped bubble on the right represents the standalone PtG facility that can buy electricity from the open market to produce hydrogen sold at a timeinvariant price. Integration of the two subsystems enables the transfer of renewable power to the PtG facility (red arrow). Such integration will generally entail operational gains if the buying price of power exceeds the selling price faced by the renewable source. Any operational gains from vertical integration have to be traded-off against the investment cost of capacity if one or both subsystems are not profitable on their own. Our analysis examines this trade-off through the lens of an investor who seeks to maximize the NPV of the integrated energy system by optimally sizing the relative capacity of the two subsystems.

\subsection{Contribution Margins}

For given capacity investments, the integrated system shown in Figure 1 will seek to maximize the periodic contribution margin by optimizing the use of the available capacity in real time. The key variables in 
this optimization are the amount of power the renewable system produces at a particular point in time and the corresponding prices at which electricity can be bought and sold externally.

Let $p^{s}(t)$ denote the selling price per kilowatt hour $(\mathrm{kWh})$ at which renewable energy can be sold on the open market at time $t$. For modeling purposes, we view time as a continuous variable $t$ ranging from 0 to 8,760 hours. The magnitude and intertemporal distribution of prices are assumed to be constant across the $T$ years of the facility. We denote by $k_{e}$ the peak capacity in kilowatt $(\mathrm{kW})$ of the renewable energy source and by $C F(t)$ the capacity factor at time $t$. The capacity factor is a scalar between 0 and 1 reflecting the actual percentage of the maximum power the system can generate. ${ }^{6}$ Thus, $C F(t) \cdot k_{e}$ represents the actual amount of power generated at time $t$, corresponding to an investment in $k_{e} \mathrm{~kW}$ of peak capacity.

Let $p^{b}(t)$ denote the price per $\mathrm{kWh}$ that would have to be paid for electricity procured on the open market at time $t$. During hours when electricity trades at a positive price, we posit the no-arbitrage condition that $p^{b}(t) \geq p^{s}(t)$. This condition is descriptive of most electricity markets. Furthermore, wholesale electricity markets increasingly exhibit patterns where at certain hours surplus electricity is unloaded on the grid and therefore prices become negative. Our analysis assumes that the renewable energy subsystem can be idled at no cost, as is generally possible for both wind and solar electricity generation. Instead of an explicit option to curtail production whenever prices turn negative, we specify equivalently that renewable power is always produced at full capacity but can be disposed off at no charge $\left(p^{s}(t)=0\right)$, whenever the buying prices turn negative. Formally, we assume:

$$
p^{s}(t)\left\{\begin{array}{cc}
\leq p^{b}(t) & \text { if } p^{b}(t) \geq 0 \\
=0 & \text { if } p^{b}(t)<0
\end{array}\right.
$$

Given supply of electricity from either the external market or the internal renewable source, the conversion value per kilogram of gas (hydrogen) produced is the selling price of the gas minus the variable operating costs. These costs include water and other variable consumable inputs like those used to deionize the water. We denote by $p_{h}$ the price per kilogram $(\mathrm{kg})$ of the gas and by $w_{h}$ the variable operating cost per $\mathrm{kg}$ produced. The conversion rate of the $\mathrm{PtG}$ process (in $\mathrm{kg} / \mathrm{kWh}$ ) is represented by the parameter $\eta$, reflecting the amount of gas that can be produced from $1 \mathrm{kWh}$ of electricity. Accordingly, the conversion value is given by:

$$
C V_{h}=\eta \cdot\left(p_{h}-w_{h}\right)
$$

For a stand-alone PtG system based entirely on electricity purchased on the open market, the contribution margin obtained at time $t$ would therefore be:

$$
C M\left(t \mid k_{h}\right)=\left[C V_{h}-p^{b}(t)\right] \cdot k_{h},
$$

if the PtG system has the capacity to absorb $k_{h} \mathrm{~kW}$ of power at any point in time.

The contribution margin that can be attained from a vertically integrated system accumulates in four different phases that differ in terms of electricity prices and the conversion value of hydrogen. In Phase 1 of the diagram in Figure 2, both the buying and the selling electricity price exceed the conversion value: $p^{b}(t) \geq p^{s}(t) \geq C V_{h} \geq 0$. As a consequence, the plant operator will keep the PtG facility idle. Since the variable operating cost of the renewable energy source is negligible, the entire electricity generation capacity will be fully exhausted and the contribution margin of the vertically integrated energy system is equal to:

$$
C M_{1}\left(t \mid k_{e}\right)=p^{s}(t) \cdot C F(t) \cdot k_{e} .
$$

In Phase 2, the buying price exceeds the conversion value of hydrogen, which, in turn, exceeds the selling price: $p^{b}(t) \geq C V_{h}>p^{s}(t) \geq 0$. It is then preferable to convert the generated renewable energy without further purchases from the external electricity market. Since the electrolyzer of the PtG plant can absorb renewable electricity up to its peak capacity, $k_{h}$, we introduce the notation $z\left(t \mid k_{e}, k_{h}\right)$ to capture the effective conversion capacity at time $t$. This capacity is the minimum of the capacity factor of the renewable energy source and the peak capacity of the PtG plant, and represents the $\mathrm{kW}$ of electricity that the PtG processing facility can receive and absorb internally from the renewable source at time $t$ :

$$
z\left(t \mid k_{e}, k_{h}\right) \equiv \min \left\{C F(t) \cdot k_{e}, k_{h}\right\} .
$$

We shall ignore switching costs associated with ramping the $\mathrm{PtG}$ facility up or down, since for most electrolyzers the associated efficiency losses can be considered negligible (Buttler and Spliethoff 2018). The contribution margin of the integrated system in Phase 2 is the contribution margin of renewable energy plus the associated conversion premium:

$$
\begin{aligned}
C M_{2}\left(t \mid k_{e}, k_{h}\right)= & p^{s}(t) \cdot C F(t) \cdot k_{e} \\
& +\left[C V_{h}-p^{s}(t)\right] \cdot z\left(t \mid k_{e}, k_{h}\right) .
\end{aligned}
$$

In Phase 3, both electricity prices are non-negative and less than the conversion value of gas: $C V_{h}>p^{b}(t) \geq p^{s}(t) \geq 0$. It is then optimal to convert the generated renewable energy and buy electricity from the market to fully utilize the remaining $\mathrm{PtG}$ capacity. The attainable contribution margin is then 
Figure 2 Phase Diagram [Color figure can be viewed at wileyonlinelibrary.com]

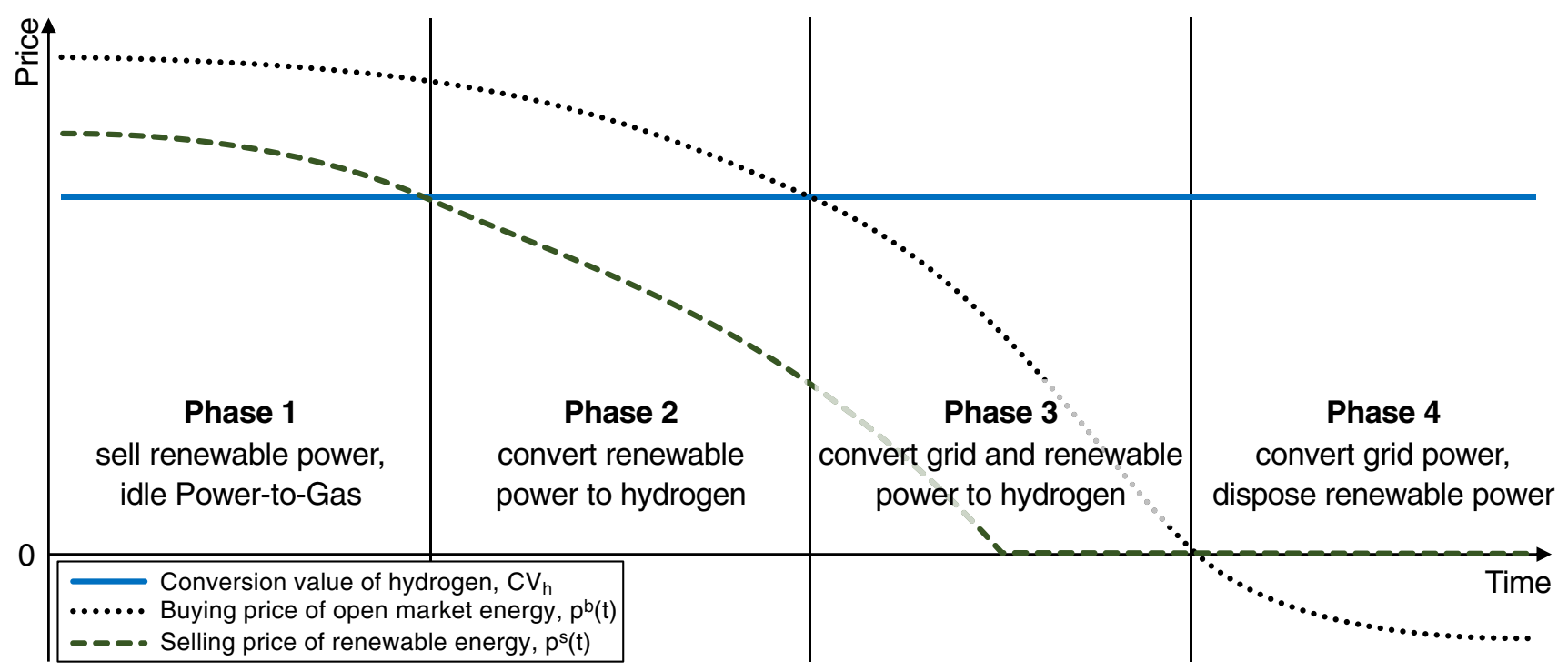

the sum of both stand-alone energy systems plus the conversion premium of renewable energy:

$$
\begin{aligned}
C M_{3}\left(t \mid k_{e}, k_{h}\right)= & p^{s}(t) \cdot C F(t) \cdot k_{e} \\
& +\left[C V_{h}-p^{s}(t)\right] \cdot z\left(t \mid k_{e}, k_{h}\right) \\
& +\left[C V_{h}-p^{b}(t)\right] \cdot\left[k_{h}-z\left(t \mid k_{e}, k_{h}\right)\right] .
\end{aligned}
$$

Finally, in Phase 4, the buying price is negative and thus $C V_{h} \geq p^{s}(t)=0>p^{b}(t)$. The facility will then idle the renewable energy source and exhaust the PtG capacity with negatively priced electricity from the market. Accordingly, the contribution margin in that scenario equals:

$$
C M_{4}\left(t \mid k_{h}\right)=\left[C V_{h}-p^{b}(t)\right] \cdot k_{h} .
$$

For further reference, we note that in a stationary environment where prices and output are constant, the contribution margin of a vertically integrated energy system corresponds to just one of the four phases illustrated in Figure 2. With time-varying prices and capacity factors, the optimized contribution margin of a vertically integrated energy system can be expressed as follows. ${ }^{7}$

LEMMA 1. The optimized contribution margin of a vertically integrated energy system at time $t$ is:

$$
\begin{aligned}
C M\left(t \mid k_{e}, k_{h}\right)=p^{s}( & t) \cdot C F(t) \cdot k_{e} \\
+ & {\left[p^{b+}(t)-p^{b}(t)\right] \cdot k_{h} } \\
& +\left[p^{+}(t)-p^{s}(t)\right] \cdot z\left(t \mid k_{e}, k_{h}\right),
\end{aligned}
$$

where $p^{b+}(t) \equiv \max \left\{p^{b}(t), C V_{h}\right\}$ and $p^{+}(t) \equiv \max \{\min$ $\left.\left\{p^{b}(t), C V_{h}\right\}, p^{s}(t)\right\}$.
Lemma 1 shows that the contribution margin of a vertically integrated energy system can be expressed as the sum of the contribution margins of the two stand-alone energy systems plus a third term that captures the economic interaction of the two subsystems. The term $p^{b+}(t)-p^{b}(t)=\max \left\{C V_{h}-p^{b}(t), 0\right\}$ will be referred to as the conversion premium of hydrogen. It reflects the option for the stand-alone PtG system to idle the electrolyzer at times when the buying price of electricity exceeds the conversion value of hydrogen. The final term of (9) reflects potential synergies, that is, the benefit of consuming the intermediate input internally. We refer to

$$
p^{+}(t)-p^{s}(t)=\max \left\{\min \left\{p^{b}(t), C V_{h}\right\}-p^{s}(t), 0\right\}
$$

as the price premium of the vertically integrated energy system at time $t$. As shown in the following sections, a positive price premium, that is, $p^{+}(t)-p^{s}(t)>0$ for some $t$, is necessary but generally not sufficient for a vertically integrated system to generate a NPV that exceeds the sum of the optimized values of the two stand-alone systems.

\subsection{Net Present Values}

A vertically integrated energy system yields cash inflows in the form of optimized contribution margins. Such a system will create value if the discounted sum of the cash inflows collectively covers the initial cash outflow for capacity investments plus the subsequent periodic operating costs, including corporate income taxes. To identify conditions for the emergence of a synergistic investment value by the vertically integrated systems, it will prove useful to express the overall NPV in terms of unit costs and revenues. Specifically, we build on the definition 
of the Levelized Cost of Electricity (LCOE), a common unit cost measure for stand-alone electricity generation systems; see, for instance, Islegen and Reichelstein $(2011){ }^{8}$

The LCOE aggregates all costs occurring over the lifetime of a power plant to deliver one unit of electricity output. The LCOE of a one $\mathrm{kW}$ facility can be expressed as:

$$
L C O E=w_{e}+f_{e}+\Delta \cdot c_{e} .
$$

Here, the subscript $e$ stands for electricity; $w$ represents the variable operating cost per $\mathrm{kWh}, f$ the levelized fixed operating cost per $\mathrm{kWh}, c$ the levelized capacity cost of the facility per $\mathrm{kWh}$, and, finally, $\Delta$ the tax factor covering the impact of income taxes and the depreciation tax shield. Since the variable operating cost for wind and solar power is negligible, we set $w_{e}=0$. The LCOE is determined by the following underlying parameters: the system price, $S P_{e}$, for the generation capacity (in $\$$ per $\mathrm{kW}$ ), the fixed operating cost in year $i, F_{e i}$ (in $\$$ per $\mathrm{kW}$ ), a discount factor, $\gamma$, based on the cost of capital $r$ such that $\gamma=\frac{1}{1+r}$ (scalar). Additional parameters are the useful economic life, $T$ (in years), and system degradation factor in year $i, x^{i-1}$ (scalar).

Time-of-use electricity prices are frequently measured on an hourly basis. We denote by $m=24.365=8,760$ the number of hours per year. The discount factor $\gamma$ is based on an underlying cost of capital (interest rate) $r$. This cost of capital should be interpreted as the weighted average cost of capital (WACC) if the project is financed through both equity and debt (Islegen and Reichelstein 2011). The scalar $x$, with $0<x<1$, denotes the system degradation factor, so that $x^{i-1}$ represents the fraction of the initial capacity that is still operating in year $i$. For notational simplicity, we assume that prices and all operational parameters, except for the system degradation factor, are identical across years. The standard definition of the LCOE ignores the hourly fluctuation in capacity utilization and instead refers to the average capacity factor, $C F$, that is the average of all hourly capacity factors: $C F=\frac{1}{m} \int_{0}^{m} C F(t) d t$.

To obtain the levelized capacity cost per $\mathrm{kWh}$, the system price per $\mathrm{kW}$ is divided by the total discounted number of $\mathrm{kWh}$ that the system produces over its useful life:

$$
c_{e}=\frac{S P_{e}}{C F \cdot L} \text {. }
$$

We refer to $L \equiv m \cdot \sum_{i=1}^{T} x^{i-1} \cdot \gamma^{i}$ as the levelization factor which expresses the discounted number of hours that are available from the facility over its entire lifetime.
Similar to the levelized cost of capacity, we define the levelized fixed operating cost per $\mathrm{kWh}$ as the total discounted fixed costs that are incurred over the lifetime of the facility divided by the levelization factor adjusted by the capacity factor.

$$
f_{e}=\frac{\sum_{i=1}^{T} F_{e i} \cdot \gamma^{i}}{C F \cdot L} .
$$

To complete the formulation of the LCOE, we include corporate income taxes and the depreciation tax shield. Depreciation charges for tax purposes and interest payments on debt reduce taxable earnings. The effect of the debt tax shield is already accounted for, if the cost of capital, $r$, is viewed as a WACC. Let $d_{i}$ denote the allowable tax depreciation rate in year $i$ and $\alpha$ the effective corporate income tax rate. The useful life of renewable power plants for tax purposes is usually shorter than their useful economic life. Therefore, the tax depreciation charges are set to zero $\left(d_{i}=0\right)$ for the remaining years. The tax factor is then given by:

$$
\Delta=\frac{1-\alpha \cdot \sum_{i=1}^{T} d_{i} \cdot \gamma^{i}}{1-\alpha} .
$$

It is readily verified that $\Delta$ is increasing and convex in the tax rate $\alpha . \Delta$ exceeds one in the absence of tax credits and is bounded above by $1 /(1-\alpha)$. Because of the time value of money, an accelerated tax depreciation schedule reduces $\Delta$. If the tax code allows for a full write-off immediately $\left(d_{0}=1\right.$ and $d_{i}=0$ for $i>0$ ), the tax factor equals one.

Some countries, including the United States, grant subsidies in form of a tax credit for renewable energy production. For wind power, this takes the form of a PTC per kWh of electricity produced (U.S. Department of Energy 2016) as:

$$
p t c=\frac{\sum_{i=1}^{T} \mathrm{PTC}_{i} \cdot x^{i-1} \cdot \gamma^{i}}{(1-\alpha) \sum_{i=1}^{T} x^{i-1} \cdot \gamma^{i}},
$$

where $P T C_{i}$ denotes the tax credit of year $i$. Since the duration of the PTC is generally shorter than the useful life of wind turbines, we set $P T C_{i}=0$ for the remaining years. The credit adds to the after-tax cash flow and is therefore divided by $(1-\alpha)$. Overall, the LCOE in the presence of PTCs can be expressed as $L C O E=w_{e}+f_{e}+\Delta \cdot c_{e}-p t c$.

On the revenue side, the capacity factor, $C F(t)$ and the attainable revenue at time $t, p^{s}(t)$, vary in real time. Accordingly, we denote by $\varepsilon(t)$ the multiplicative deviation of $C F(t)$ from its average value $C F$ and 
by $\mu(t)$ the multiplicative deviation of $p^{s}(t)$ from the average selling price, $p^{s}$ :

$$
\epsilon(t)=\frac{C F(t)}{C F} \text { and } \mu(t)=\frac{p^{s}(t)}{p^{s}}
$$

By definition:

$$
\frac{1}{m} \int_{0}^{m} \epsilon(t) d t=\frac{1}{m} \int_{0}^{m} \mu(t) d t=1 .
$$

In the terminology of Reichelstein and Sahoo (2015), the co-variation coefficient captures the variation between output and price:

$$
\Gamma^{\mathcal{S}} \equiv \frac{1}{m} \int_{0}^{m} \epsilon(t) \cdot \mu(t) d t .
$$

Clearly, the co-variation coefficient is non-negative and zero only if the renewable energy source generates electricity exclusively at times when prices are zero. For a dispatchable energy source with $C F(t) \equiv C F$, we obtain $\Gamma^{s}=1$. Similarly, $\Gamma^{s}=1$ if $p^{s}(t) \equiv p^{s}$. As one would expect, the profit margin of the renewable energy source improves if more power is generated during times of peak power prices as reflected in a larger co-variation coefficient.

The stand-alone NPV of an intermittent power generation system is then given by:

$$
N P V\left(k_{e}\right)=(1-\alpha) \cdot L \cdot\left(\Gamma^{s} \cdot p^{s}-L C O E\right) \cdot C F \cdot k_{e} .
$$

We refer to $\Gamma^{s} \cdot p^{s}-L C O E$ as the (pre-tax) profit margin per $\mathrm{kWh}$ for the renewable energy source. If this margin is positive, intermittent power generation is said to be cost competitive on its own. Consistent with Reichelstein and Sahoo (2015), Equation (18) shows that a renewable electricity generation system is cost competitive (yields a positive NPV) in an environment with time varying prices if the average sales price adjusted by the co-variation coefficient exceeds the levelized cost of electricity.

For the hydrogen subsystem, our definition of the conversion value of hydrogen, $C V_{h}$, already incorporates the variable operating costs of converting electricity and water into hydrogen. For investment purposes, the additional relevant cost then is the Levelized Fixed Cost of Hydrogen ( $L F C H)$. On a life-cycle basis, it captures the capacity and fixed operating costs per kWh required to absorb electricity at the PtG plant (Farhat and Reichelstein 2016). With the subscript $h$ representing hydrogen, the levelized cost of electricity is given:

$$
L F C H=f_{h}+\Delta \cdot c_{h},
$$

where: ${ }^{9}$

$$
c_{h}=\frac{S P_{h}}{L}, \quad f_{h}=\frac{\sum_{i=1}^{T} F_{h i} \cdot \gamma^{i}}{L} .
$$

To express the NPV of a PtG facility, we introduce the average conversion premium:

$$
p^{b+}-p^{b} \equiv \frac{1}{m} \int_{0}^{m}\left[p^{b+}(t)-p^{b}(t)\right] d t .
$$

The NPV of a stand-alone PtG facility can then be stated as:

$$
N P V\left(k_{h}\right)=(1-\alpha) \cdot L \cdot\left(p^{b+}-p^{b}-L F C H\right) \cdot k_{h},
$$

with $p^{b+}-p^{b}-L F C H$ representing the unit profit margin of PtG. PtG will be referred to as cost competitive (profitable) if this margin is positive.

Similar to the covariance between output and selling price for renewable electricity, we need to capture the co-variation between hydrogen output and the price premium, $p^{+}(t)-p^{s}(t)$ of a vertically integrated energy system. Accordingly, we let $\mu^{+}(t)$ denote the multiplicative deviation factor of the price premium of the integrated energy system from the average premium of an integrated energy system, $p^{+}-p^{s}$ at time $t$ :

$$
\mu^{+}(t)=\frac{p^{+}(t)-p^{s}(t)}{p^{+}-p^{s}} .
$$

As before, the multiplicative deviation factor reflects a normalization so that $\frac{1}{m} \int_{0}^{m} \mu^{+}(t)=1$. Finally, we introduce $z\left(k_{e}, k_{h}\right)$ as follows:

$$
z\left(k_{e}, k_{h}\right) \equiv \frac{1}{m} \int_{0}^{m} z\left(t \mid k_{e}, k_{h}\right) \cdot \mu^{+}(t) d t .
$$

Proposition 1. The NPV of the vertically integrated energy system of size $\left(k_{e}, k_{h}\right)$ is:

$$
\begin{aligned}
N P V\left(k_{e}, k_{h}\right)= & (1-\alpha) \cdot L \cdot\left[\left(\Gamma^{s} \cdot p^{s}-L C O E\right) \cdot C F \cdot k_{e}\right. \\
& +\left(p^{b+}-p^{b}-L F C H\right) \cdot k_{h} \\
& \left.+\left(p^{+}-p^{s}\right) \cdot z\left(k_{e}, k_{h}\right)\right] .
\end{aligned}
$$

The first two terms of the NPV expression in Equation (24) reflect the value created by the two stand-alone systems. The third term captures the time-averaged synergistic gains. These gains are a function of both the average price premium $\left(p^{+}-p^{s}\right)$ and the effective (time-averaged) conversion capacity $z\left(k_{e}, k_{h}\right)$, which, in turn, is determined by the relative size of the two subsystems. 
An immediate consequence of Proposition 1 is that if both stand-alone systems are profitable on their own, a vertically integrated energy system will generate synergies unless $p^{+}=p^{s}$. On the other hand, if either one or both of the stand-alone systems exhibit a negative NPV, then the "synergistic" third term in Equation (24) would have to compensate for the losses associated with the stand-alone system. Formally, a vertically integrated energy system is said to have synergistic investment value if for some combination $\left(k_{e}, k_{h}\right)$ :

$$
\begin{aligned}
N P V\left(k_{e}, k_{h}\right) & >\max \left\{N P V\left(k_{e}, 0\right), 0\right\} \\
& +\max \left\{N P V\left(0, k_{h}\right), 0\right\} .
\end{aligned}
$$

We note that if the inequality in Equation (25) is met for some $\left(k_{e}, k_{h}\right)$, there is no upper bound on the attainable NPV in our model because the function $N P V\left(k_{e}, k_{h}\right)$ is homogeneous of degree 1 , that is, $N P V\left(\theta \cdot k_{e}, \theta \cdot k_{h}\right)=\theta \cdot N P V\left(k_{e}, k_{h}\right)$ for any $\theta>0$.

\section{Synergistic Investment Value}

The vertically integrated system may exhibit synergistic investment value in each of the four possible scenarios that arise depending on whether the two stand-alone systems are cost competitive on their own, or not. If indeed both subsystems are profitable on their own, one would expect a synergistic investment value that results from being able to by-pass the electricity market and thereby avoiding the mark-up between selling and buying prices for electricity. For completeness, we state the following formal result.

Corollary to Proposition 1. If both stand-alone energy systems are cost competitive on their own, the vertically integrated energy system has synergistic investment value if and only if for some $t \in[0,8760]$ :

$$
\min \left\{p^{b}(t), C V_{h}\right\}>p^{s}(t) .
$$

Clearly, the inequality in Equation (26) can only hold during time intervals that correspond to Phases 2 or 3 in Figure 2. By Proposition 1, a synergistic investment value hinges entirely on $p^{+}-p^{s}>0$. This inequality will hold unless for all $t: \min \left\{p^{b}(t), C V_{h}\right\} \leq p^{s}(t) .{ }^{10} \mathrm{We}$ note that in a hypothetical stationary environment, where prices and output generation are time-invariant, there will always be a synergistic investment value if both stand-alone systems are profitable on their own, provided $C V_{h}>p^{b}>p^{s}$.

We next turn to the two mixed cases in terms of cost competitiveness of the two stand alone systems. Similar to the co-variation factor $\Gamma^{s}$, we denote by $\Gamma^{+}$the co-variation coefficient between the renewable power capacity factors and the real-time price premia associated with PtG conversion:

$$
\Gamma^{+}=\frac{1}{m} \int_{0}^{m} \epsilon(t) \cdot \mu^{+}(t) d t
$$

\section{PROPOSITION 2.}

(i) Suppose renewable power is cost competitive $\left(\Gamma^{s} \cdot p^{s}-L C O E>0\right)$, while stand-alone PtG is not $\left(p^{b+}-p^{b}-L F C H<0\right)$. The vertically integrated energy system then has synergistic investment value if and only if:

$$
p^{+}-p^{s}>L F C H-\left(p^{b+}-p^{b}\right) .
$$

(ii) Suppose stand-alone PtG is cost competitive, but renewable power generation is not. The vertically integrated energy system then has synergistic investment value if and only if:

$$
\Gamma^{+} \cdot\left(p^{+}-p^{s}\right)>\operatorname{LCOE}-\Gamma^{s} \cdot p^{s}
$$

Condition (28) in Proposition 2 states that the average price premium associated with PtG conversion must exceed the negative profit margin associated with the PtG system. The average revenue and life cycle cost associated with power generation are irrelevant since that activity will be undertaken regardless of the vertical integration decision. If the inequality in Equation (28) were to hold barely, the optimal PtG capacity would be small relative to the size of the renewable power source. To see this, suppose the capacity of the renewable power source is normalized to $k_{e}=1$. Differentiating the expression for $\operatorname{NPV}\left(1, k_{h}\right)$ in $k_{h}$, we note that this partial derivative is zero at the unique point $k_{h}^{*}\left(k_{e}=1\right)$ given as the solution to the equation:

$$
\frac{\partial}{\partial k_{h}} z\left(1, k_{h}^{*}(1)\right) \cdot\left(p^{+}-p^{s}\right)=L F C H-\left(p^{b+}-p^{b}\right),
$$

where

$$
\frac{\partial}{\partial k_{h}} z\left(1, k_{h}\right)=\frac{1}{m} \int_{\left\{t \mid k_{h} \leq C F(t)\right\}} \mu^{+}(t) d t .
$$

Clearly, $\frac{\partial}{\partial k_{h}} z\left(1, k_{h}\right)$ is decreasing in $k_{h}$ with $\lim _{k_{h} \rightarrow 0} \frac{\partial}{\partial k_{h}} z\left(1, k_{h}\right)=1$, thus identifying the inequality in Equation (28) as necessary and sufficient for an integrated system to have synergistic investment value.

An analogous argument verifies the necessity and sufficiency of the inequality in Equation (29) for the presence of a synergistic investment value if the PtG facility is profitable on its own, but renewable electricity generation is not. Holding the size of the 
electrolyzer fixed at $k_{h}=1$, we obtain the corresponding optimal size of $k_{e}^{*}\left(k_{h}=1\right)$ as the unique solution to the equation:

$$
\frac{\partial}{\partial k_{e}} z\left(k_{e}^{*}(1), 1\right) \cdot\left(p^{+}-p^{s}\right)=\left(L C O E-\Gamma^{s} \cdot p^{s}\right) \cdot C F,
$$

where

$$
\frac{\partial}{\partial k_{e}} z\left(k_{e}, 1\right)=\frac{1}{m} \int_{\left\{t \mid C F(t) \cdot k_{e}<1\right\}} \mu^{+}(t) \cdot C F(t) d t .
$$

The uniqueness of $k_{e}^{*}(1)$ follows from the fact that $\frac{\partial}{\partial k_{e}} z\left(k_{e}, 1\right)$ is decreasing in $k_{e}$ such that $\lim _{k_{e} \rightarrow 0} \frac{\partial}{\partial k_{e}} z\left(k_{e}, 1\right)=C F \cdot \Gamma^{+}$and $\lim _{k_{e} \rightarrow \infty} \frac{\partial}{\partial k_{e}} z\left(k_{e}, 1\right)=0$.

Overall, the $\operatorname{NPV}(\cdot, \cdot)$ function is concave in $\left(k_{e}, k_{h}\right){ }^{11}$ If (28) holds, $N P V\left(k_{e}, \cdot\right)$ is a single-peaked function of $k_{h}$, while if (29) holds, $\operatorname{NPV}\left(\cdot, k_{h}\right)$ is a single-peaked function of $k_{e}$. Furthermore, the fact that $N P V\left(k_{e}, k_{h}\right)$ is homogeneous of degree 1 implies that, conditional on (28) being satisfied, the conditional maximizer $k_{h}^{*}(\cdot)$ will be a linear function of $k_{e}$. Figure 3 illustrates this relationship.

In a hypothetical stationary environment where power prices and power generation are time-invariant, we find if there is synergistic investment value in either one of the two scenarios identified in Proposition 2, the optimally sized vertically integrated system will be such that all renewable power is consumed internally. For scenario (i) in Proposition 2, (28) simplifies to $C V_{h}-p^{s}>L F C H$, provided hydrogen conversion is valuable in the first place, that is, $C V_{h}>p^{b}>p^{s}$. It will then be optimal to size the PtG facility such that $k_{h}^{*}\left(k_{e}\right)=C F \cdot k_{e}$ and all renewable energy will be consumed internally. For scenario (ii) in Proposition 2, (28) simplifies to $\max \left\{p^{b}, C V_{h}\right\}>$ $L C O E$. It would then be optimal to size the renewable energy source such that $C F \cdot k_{e}^{*}\left(k_{h}\right)=k_{h}$ and all renewable energy will again be consumed internally.

If neither stand-alone subsystem is cost competitive on its own, an investor might still be willing to acquire a combination of the two subsystems

Figure 3 Linearity of the Optimal PtG Capacity Size [Color figure can be viewed at wileyonlinelibrary.com]

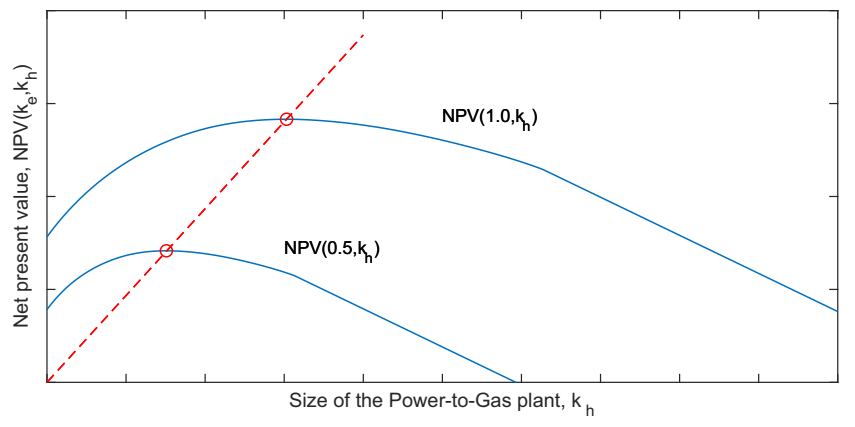

provided the synergistic investment value more than compensates for the losses associated with the two stand-alone systems. Figure 4 illustrates this possibility. Without loss of generality, we again anchor the size of the two subsystems, such that $k_{e}=1$ and $k_{h}$ is chosen optimally at $k_{h}^{*}\left(k_{e}=1\right)$ to maximize:

$$
\left(p^{+}-p^{s}\right) \cdot z\left(1, k_{h}\right)+\left(p^{b+}-p^{b}-L F C H\right) \cdot k_{h} .
$$

As argued in connection with Proposition 2, $k_{h}^{*}(1)>0$ if and only if $p^{+}-p^{s}>L F C H-\left(p^{b+}-p^{b}\right)$.

PROPOSITION 3. If neither PtG nor intermittent renewable power is cost competitive on its own, a necessary and sufficient condition for a vertically integrated energy system to have synergistic investment value is that:

$$
\begin{gathered}
\left(p^{+}-p^{s}\right) \cdot z\left(1, k_{h}^{*}(1)\right)+\left(p^{b+}-p^{b}-L F C H\right) \cdot k_{h}^{*}(1) \\
\quad+\left(\Gamma^{s} \cdot p^{s}-L C O E\right) \cdot C F>0 .
\end{gathered}
$$

While the necessary and sufficient condition for synergies identified in Equation (34) is stated in terms of the endogenously optimized value $k_{h}^{*}$, we obtain the following weaker necessary condition in terms of the average price premia, the $\mathrm{LFCH}$ and the unit profit margin of the renewable energy source.

Corollary to Proposition 3. Suppose neither PtG nor intermittent renewable power is cost competitive on its own. The inequality:

$p^{+}-p^{s}+p^{b+}-p^{b}-L F C H+\left(\Gamma^{s} \cdot p^{s}-L C O E\right) \cdot C F>0$

then provides a necessary condition for the vertically integrated energy system to have synergistic investment value.

The preceding claim is a direct consequence of Proposition 3 because, by construction, both $k_{h}^{*}$ and $z\left(1, k_{h}^{*}(1)\right)$ are less than one. Finally, the inequality in Equation (35) simplifies to $C V_{h}>L F C H+L C O E$ for a stationary environment with constant prices and output. Thus, the synergistic investment value of the vertically integrated system in a stationary environment hinges entirely on its levelized cost and the conversion value of hydrogen. The corresponding optimal size for PtG is such that $k_{h}=C F \cdot k_{e}$, so that hydrogen is produced only from internally generated renewable electricity.

\section{Application: Wind Energy and Power-to-Gas}

\subsection{Stand-Alone Wind Energy}

We now apply the preceding model framework to vertically integrated energy systems that combine 
Figure 4 Synergistic Investment Value if Neither Stand-Alone Energy System is Cost Competitive on its Own [Color figure can be viewed at wileyon linelibrary.com]

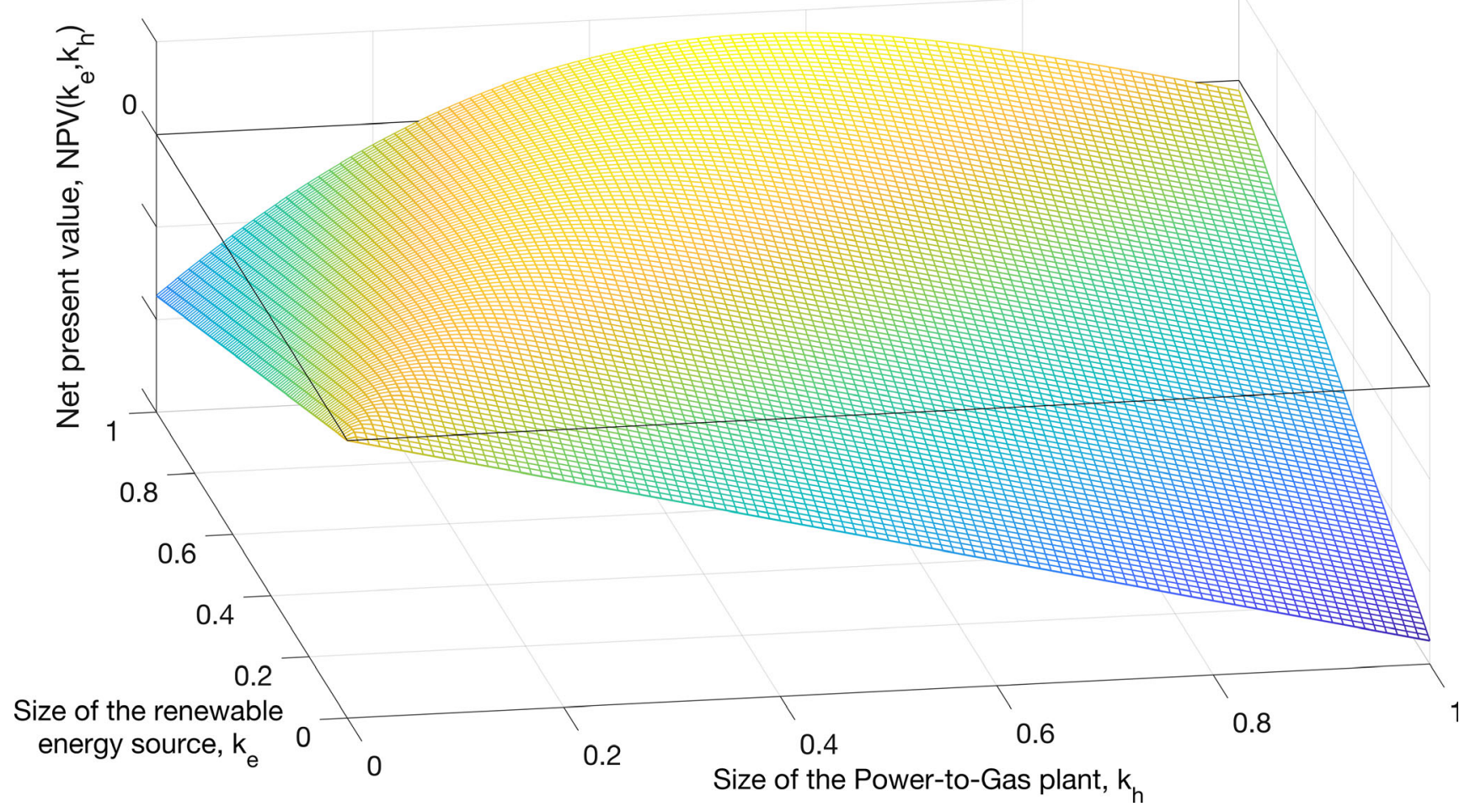

wind power with PtG. Our numerical analysis focuses on Germany and Texas, two jurisdictions that have deployed considerable amounts of wind power in recent years. Wind energy naturally complements $\mathrm{PtG}$ as wind power tends to reach peak production levels at night when demand from the grid and electricity prices are relatively low (Reichelstein and Sahoo 2015, Wozabal et al. 2016). We base our initial calculations on 2017 data. Our data inputs are obtained from journal articles, industry reports, publicly available reports and interviews with industry sources (see the Appendix for a comprehensive list).

Wind energy is eligible for a federal PTC in the United States. It is paid per $\mathrm{kWh}$ of electricity generated (U.S. Department of Energy 2016). As shown in section 2, the PTC can be levelized and then effectively be subtracted from the LCOE. Beginning in 2017, Germany replaced its traditional fixed feed-in premium for wind energy with a competitive auction system in which successful bidders are guaranteed a minimum price per $\mathrm{kWh}$, with the government paying the difference between the successful bid and the actual revenue obtained from wind energy in the market place (EEG 2017). We refer to this difference as the Production Premium (PP). ${ }^{12}$

Table 1 summarizes the calculation of the unit profit margin for wind energy in both jurisdictions. ${ }^{13}$ The LCOE of wind energy amounts to $4.83 € \notin / \mathrm{kWh}$ in Germany. The substantially lower LCOE $2.41 \$ \phi /$ $\mathrm{kWh}$ in Texas reflects the impact of the PTC and, to a smaller extent, a higher capacity factor. The average selling prices of electricity amount to $3.46 € \notin / \mathrm{kWh}$ and $2.44 \$ \phi / \mathrm{kWh}$, respectively. The corresponding co-variation coefficients of 0.87 and 0.93 indicate that prices tend to be below their average values during periods of above average wind output. We interpret the procurement auctions in Germany as competitive and therefore the profit margins are zero by construction. Thus, we infer the PP as the difference between the winning bids and the observed selling prices adjusted by the co-variation coefficients. The estimates we obtain are corroborated by the observation that the range of observed winning bids (guaranteed selling prices) in 2017 was between 3.82 and $5.71 € \not /$ $\mathrm{kWh}$ and our independent LCOE estimate is just about in the middle of that range.

\subsection{Stand-alone Power-to-Gas}

As a producer of industry gases, a PtG facility in Germany is eligible to purchase electricity at the wholesale market price plus a relatively small markup for taxes, fees and levies. For Texas, we use the industrial rate offered by Austin Energy. Because of its grid connection, the PtG facility can also provide frequency control to the grid by rapidly absorbing excess electricity to balance supply and demand. 


\section{Table 1 Profit Margins for Wind Energy}

\begin{tabular}{lll}
\hline & Germany & Texas \\
\hline Input variables & & \\
System price, $S P_{e}$ & $1,180 € / \mathrm{kW}$ & $1,566 \$ / \mathrm{kW}$ \\
Capacity factor, CF & $30.33 \%$ & $44.39 \%$ \\
Levelized $P P$ or $P T C$ & $1.81 € \notin / \mathrm{kWh}$ & $1.31 \$ \notin / \mathrm{kWh}$ \\
Cost of capital (WACC), $r$ & $4.00 \%$ & $6.00 \%$ \\
Profit margins & & \\
Levelized cost of electricity, LCOE & $4.83 € \notin / \mathrm{kWh}$ & $2.42 \$ \notin / \mathrm{kWh}$ \\
Selling price of electricity, $p^{s}$ & $3.46 € \notin / \mathrm{kWh}$ & $2.44 \$ \notin / \mathrm{kWh}$ \\
Co-variation coefficient, $\Gamma^{S}$ & 0.87 & 0.93 \\
Profit margin & $0.00 € \notin / \mathrm{kWh}$ & $-0.15 \$ \notin / \mathrm{kWh}$ \\
\hline
\end{tabular}

Incorporating the revenues from frequency control into the price at which the facility can purchase electricity, the buying price of electricity averages $3.93 €$ $\phi / \mathrm{kWh}$ in Germany and $5.39 \$ \phi / \mathrm{kWh}$ in Texas (see the Appendix for details).

A PtG facility could be installed onsite or adjacent to a hydrogen customer. ${ }^{14}$ The observed market prices for hydrogen are clustered in three segments that vary primarily with scale (volume) and purity. In Germany, prices for large-scale supply amount on average to $2.0 € / \mathrm{kg}$, for medium-scale to about 3.5 $€ / \mathrm{kg}$, and for small-scale to at least $4.0 € / \mathrm{kg}$. In Texas, large-scale hydrogen supply is priced at about 2.5 $\$ / \mathrm{kg}$, while medium- and small-scale are priced at about $4.0 \$ / \mathrm{kg}$ or above $4.5 \$ / \mathrm{kg}$, respectively (Glenk and Reichelstein 2019).

Table 2 summarizes the calculation of the unit profit margin for PtG in both jurisdictions. The $\mathrm{LFCH}$ of PtG amounts to $2.36 € \varnothing / \mathrm{kWh}$ in Germany and 2.22 $\$ \phi / \mathrm{kWh}$ in Texas. For medium-scale supply, the conversion premium of hydrogen amounts to 2.93 $€ \varnothing / \mathrm{kWh}$ in Germany and $2.67 \$ \phi / \mathrm{kWh}$ in Texas, with corresponding profit margins of $0.57 € \phi / \mathrm{kWh}$ and $0.44 \$ \phi / \mathrm{kWh}$, respectively. For large-scale hydrogen supply, the conversion premium equals $1.12 € \phi /$ $\mathrm{kWh}$ in Germany and $0.54 \$ \phi / \mathrm{kWh}$ in Texas and the corresponding profit margins are $-1.24 € \notin / \mathrm{kWh}$ and $-1.69 \$ \phi / \mathrm{kWh}$ respectively. In terms of our model, we thus have the scenarios of the Corollary to Proposition 1 or Proposition 2 in Germany depending on the scale of hydrogen sales, while the setting in Texas corresponds to either Proposition 2 or Proposition 3.

\subsection{Vertical Integration of Wind Energy and Power-to-Gas}

The hydrogen prices shown in Table 2 for Texas and Germany show that our analysis spans the four possible scenarios that can arise in terms of the stand-alone profitability of the two subsystems. Figure 5 indicates the presence or absence of a synergistic investment value for the vertically integrated PtG system. As one might expect, there is a synergistic investment value in Germany relative to the scenario of high hydrogen
Table 2 Profit Margins for Power-to-Gas

\begin{tabular}{|c|c|c|}
\hline & Germany & Texas \\
\hline \multicolumn{3}{|l|}{ Input variables } \\
\hline System price, $S P_{h}$ & $2,074 € / \mathrm{kW}$ & $1,822 \$ / \mathrm{kW}$ \\
\hline Conversion rate, $\eta$ & $0.019 \mathrm{~kg} / \mathrm{kWh}$ & $0.019 \mathrm{~kg} / \mathrm{kWh}$ \\
\hline $\begin{array}{l}\text { Buying price of } \\
\text { electricity, } p^{b}\end{array}$ & $3.93 € c / k W h$ & $5.39 \$ \phi / k W h$ \\
\hline $\begin{array}{l}\text { Medium-scale hydrogen } \\
\text { price, } p_{h}\end{array}$ & $3.50 € / \mathrm{kg}$ & $4.00 \$ / \mathrm{kg}$ \\
\hline $\begin{array}{l}\text { Large-scale hydrogen } \\
\text { price, } p_{h}\end{array}$ & $2.00 € / \mathrm{kg}$ & $2.50 \$ / \mathrm{kg}$ \\
\hline \multicolumn{3}{|l|}{ Profit margins } \\
\hline $\begin{array}{l}\text { Levelized fixed cost of } \\
\text { hydrogen, } L F C H\end{array}$ & $2.36 € \notin / k W h$ & $2.22 \$ \phi / k W h$ \\
\hline $\begin{array}{l}\text { Medium-scale conversion } \\
\text { premium, } p^{b+}-p^{b}\end{array}$ & $2.93 € \notin / k W h$ & $2.67 \$ \phi / \mathrm{kWh}$ \\
\hline Medium-scale profit margin & $0.57 € \notin / \mathrm{kWh}$ & $0.44 \$ \phi / \mathrm{kWh}$ \\
\hline $\begin{array}{l}\text { Large-scale conversion } \\
\text { premium, } p^{b+}-p^{b}\end{array}$ & $1.12 € \notin / \mathrm{kWh}$ & $0.54 \$ \phi / \mathrm{kWh}$ \\
\hline Large-scale profit margin & $-1.24 € \notin / k W h$ & $-1.69 \$ \phi / \mathrm{kWh}$ \\
\hline
\end{tabular}

prices in the medium-scale supply segment. Since both subsystems are profitable on their own in that scenario, the threshold for the presence of a synergistic investment value, that is, a conversion premium that is positive rather than zero (Corollary to Proposition 1), is indeed met.

Due to the relatively low hydrogen prices (largescale supply) in Germany, PtG exhibits a highly negative profit margin of $-1.24 € \phi / \mathrm{kWh}$ on its own. The synergistic price premium, $p^{+}-p^{s}$, at $0.42 € \notin /$ $\mathrm{kWh}$ is insufficient to compensate for the PtG losses, and thus there is no synergistic investment value. Arguably, the most surprising finding occurs for the scenario of low hydrogen prices in Texas. Despite the negative profit margins of the two stand-alone subsystems, we find that for a wind power capacity normalized to $1 \mathrm{~kW}$ the corresponding optimal size of the $\mathrm{PtG}$ facility is $k_{h}^{*}=0.27 \mathrm{~kW}$ and $z\left(1, k_{h}^{*}\right)=0.24$. The profit margin of PtG multiplied with $k_{h}^{*}$ then amounts to $-0.46 \$ \phi / \mathrm{kWh}$ and the profit margin of wind energy multiplied with the average capacity factor to $-0.07 \$ \phi / \mathrm{kWh}$. Yet, the price premium, $p^{+}-p^{s}$, at $2.24 \$ \phi / \mathrm{kWh}$ delivers a sufficiently strong synergistic effect which more than compensates for the two stand-alone losses (Proposition 3). ${ }^{15}$

To quantify the synergistic investment value of an integrated wind energy and PtG system, it will be instructive to calculate the break-even price of hydrogen. In the stand-alone production mode, this price is the minimum at which the PtG system breaks even, that is, the price $p_{h}$ for which $p^{b+}-p^{b}-L F C H=0$. In contrast, for a vertically integrated system the breakeven price of hydrogen is the lowest value of $p_{h}$ such that the inequality in Equation (25) holds as an equality. Figure 6 shows by how much the break-even price falls as a consequence of integrating the two energy systems. This drop is particularly pronounced in 
Figure 5 Synergistic Investment Value of Vertically Integrated Wind Energy and PtG System [Color figure can be viewed at wileyonlinelibrary.com]

\section{Power-to-Gas}

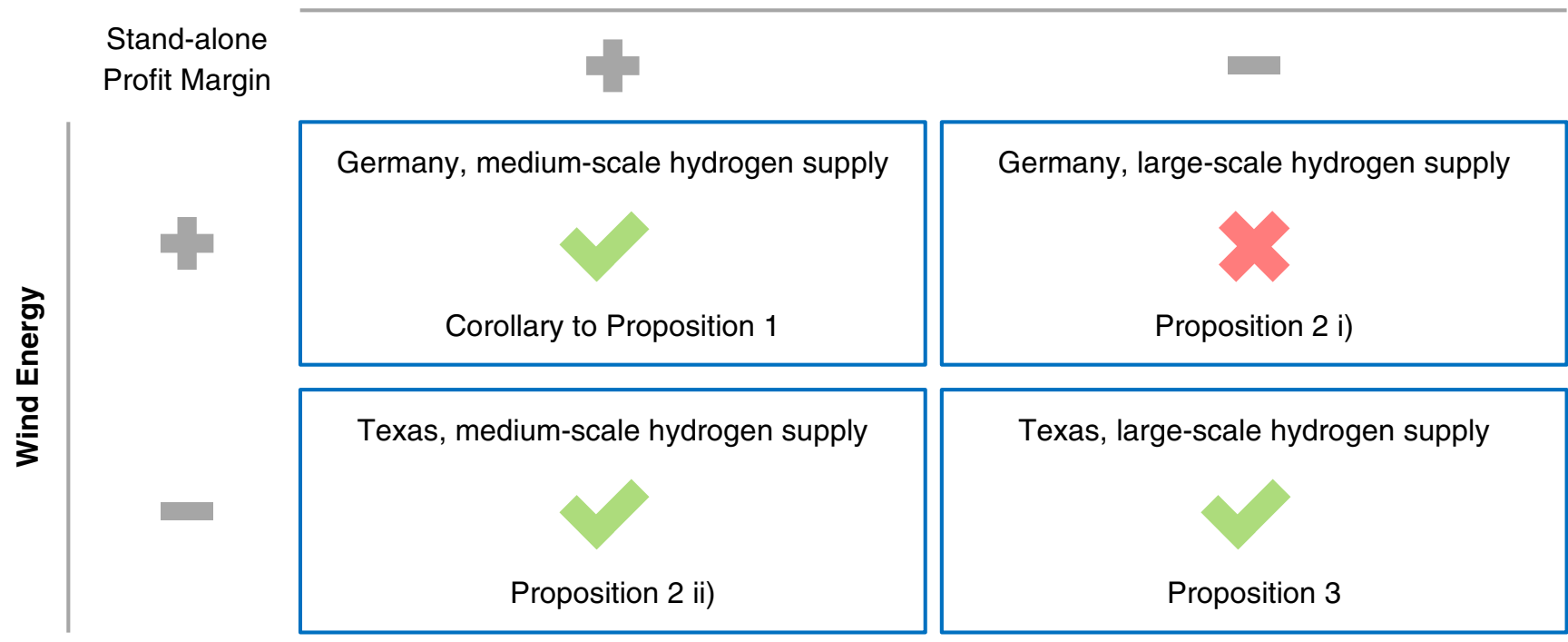

Texas where the difference between the two breakeven prices is $\$ 1.33$ per $\mathrm{kg}$, reflecting the significant price premium in Texas that yields a synergistic investment value even if both subsystems are unprofitable on their own. More broadly, the break-even values reported in Figure 6 are consistent with current market activity for early deployments of large-scale $\mathrm{PtG}$ facilities in connection with refineries and steel plants; see, for instance, Bloomberg (2017), ITM Power (2018), Voestalpine (2018), GTM (2018).

Break-even analysis can also quantify the value of giving the vertically integrated energy system access to buying electricity from the open market. Cutting off that supply branch would effectively yield a measure for the cost of renewable hydrogen, that is, hydrogen produced exclusively from wind energy. ${ }^{16}$ Figure 6 shows the break-even prices for renewable hydrogen as "renewable" prices. By construction, these prices must be higher than those of the vertically integrated system. The price difference is relatively large for Germany, indicating that in the current market environment access to the open electricity market is particularly important for the economics of hydrogen production.

To conclude this section, we solve for the optimal (relative) size of the PtG capacity for a given wind power facility the size of which has been normalized to $1 \mathrm{~kW}$. The blue lines in Figure 7 display the NPV of the vertically integrated system as a function of the size of the PtG facility for alternative hydrogen prices ranging from 1.0 to $4.0 €$ or $\$$ per $\mathrm{kg}$. Red circles mark the optimal PtG capacity size for a particular hydrogen price. Circles at $0.0 \mathrm{~kW}$ indicate that no $\mathrm{PtG}$ capacity should be installed, while a red circle at $1.0 \mathrm{~kW}$ indicates that PtG is cost competitive on its own. As demonstrated in section 3, the NPV is always a singlepeaked function of $k_{h}$ for any given hydrogen price.

In comparison to other recent studies on the economics of hydrogen, our results point to generally lower hydrogen prices (Ainscough et al. 2014, Bertuccioli et al. 2014, Felgenhauer and Hamacher 2015). We attribute this discrepancy to several factors. Most importantly, our calculations are based on vertically integrated energy systems that are sized optimally for highly capital-intensive capacity investments. In addition, our vertically integrated PtG facility is assumed to be connected to the grid and therefore obtains higher capacity utilization by converting renewable and grid electricity than it could achieve if it was to convert only renewable energy (Glenk and Reichelstein 2019). Finally, our calculations are based on most recent data reflecting the rapidly falling cost of

Figure 6 Break-Even Prices for Hydrogen Production [Color figure can be viewed at wileyonlinelibrary.com]
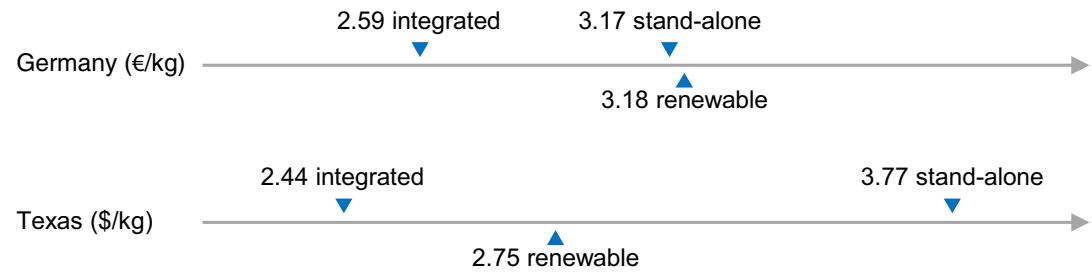
Figure 7 Optimal Power-to-Gas Capacity Size [Color figure can be viewed at wileyonlinelibrary.com]

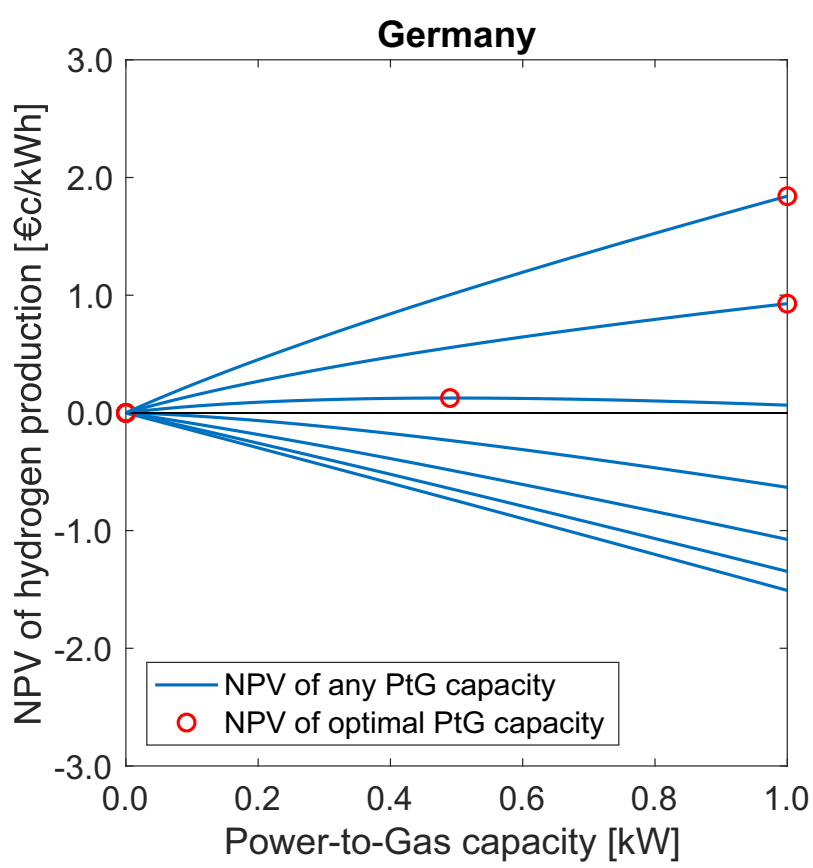

producing wind energy as well as recent changes in the acquisition cost of electrolyzers.

\subsection{Prospects for Synergistic Investment Value}

The preceding numerical findings assess the economics of wind energy combined with $\mathrm{PtG}$ on the basis of recent data. Going forward, multiple trends appear to be underway that suggest further improvements in the economics of such vertically integrated energy systems. In this subsection, we incorporate the magnitude of these trends to track changes in the break-even prices for hydrogen in future years. The break-even hydrogen prices for a vertically integrated system reported in Figure 6 are the starting points of this trajectory.

Regarding the cost structure of wind energy, we follow Wiser et al. (2016) who project that the system prices for wind turbines will decline at a rate of $4.0 \%$ per year. At the same time, these authors project an increase in the average capacity factor at an annual rate of $0.7 \%$ per year. For the acquisition cost of electrolyzers, we rely on the regression results of Glenk and Reichelstein (2019), yielding an annual 4.77\% decrease in the system price of PEM electrolyzers.

Our projections also assume that wind power in Germany and Texas will have a "driving role" in future changes of the selling prices of electricity in the wholesale market (Ketterer 2014, Paraschiv et al. 2014, Woo et al. 2011). Specifically, the difference between the LCOE in year $i, \operatorname{LCOE}(i)$, and the adjusted average selling price, $\Gamma^{s} \cdot p^{s}(i)$, is assumed to decline to zero at a constant adjustment rate such that:

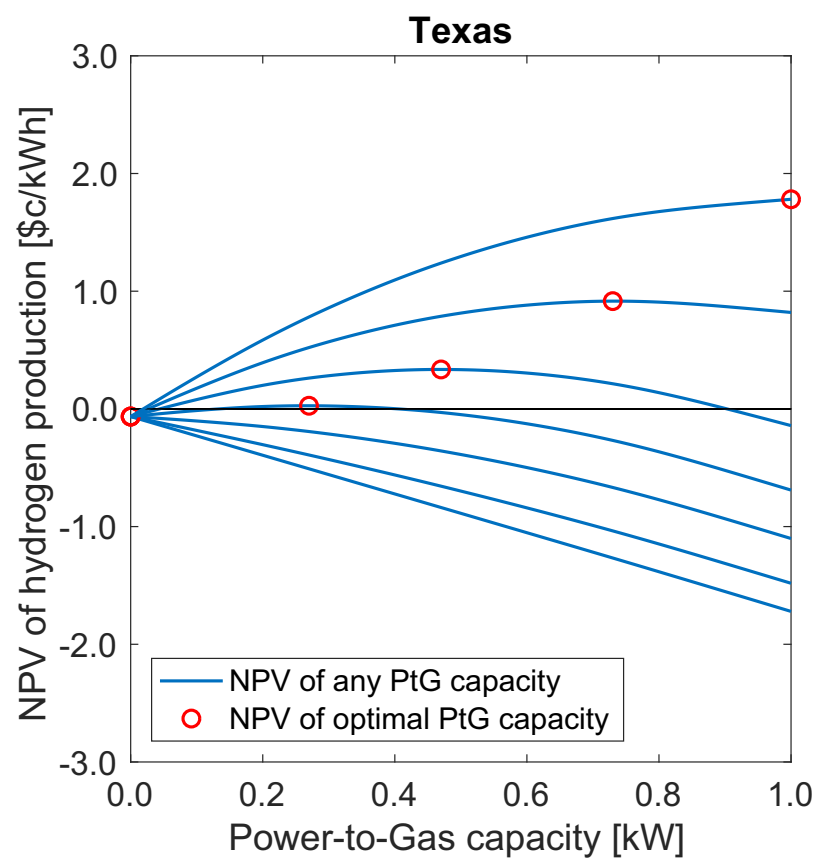

$$
\operatorname{LCOE}(i)-\Gamma^{s} \cdot p^{s}(i)=D(0) \cdot \beta^{i},
$$

where $\beta<1$ denotes the adjustment rate and $D(0) \equiv \max \left\{\operatorname{LCOE}(0)-\Gamma^{s} \cdot p^{s}(0), 0\right\}$. Since in Germany the PP is determined through a competitive auction mechanism, we expect the auction in year $i$ to yield a premium of $P P(i)=D(i)$. In Texas, our calculations anticipate the scheduled phase-out of the PTC by $20.0 \%$ per year (U.S. Department of Energy 2016) which will raise the $L C O E(i)$ for those years.

Figure 8 shows the trajectory of break-even prices for hydrogen from a vertically integrated wind power and PtG system through 2030. Specifically, the hydrogen produced in this manner is projected to become cost competitive with industrial-scale hydrogen supply, that is currently produced from fossil fuels, in the coming decade. The values shown by the solid line in Figure 8 assume an adjustment rate of $\beta=0.95$. For Texas, the hump in Figure 8 reflects the scheduled phase-out of the PTC. The values covered by the areas shaded in blue illustrate the impact of slower and faster adjustment rates ranging from 0.975 to 0.925 .

Finally, we seek to capture the idea that further increases in renewable energy are likely to increase the variance in daily and seasonal electricity prices. As noted in section 2, higher operational volatility will generally tend to accentuate the synergistic investment value of a vertically integrated system. We incorporate the possibility of increased volatility in the selling price of electricity by assuming that $p^{s}(t)$ increases by $\xi \%$ whenever $p^{s}(t)$ exceeds the average $p^{s}$ and that $p^{s}(t)$ decreases by a corresponding 
Figure 8 Trajectory of Future Hydrogen Break-Even Prices [Color figure can be viewed at wileyonlinelibrary.com]

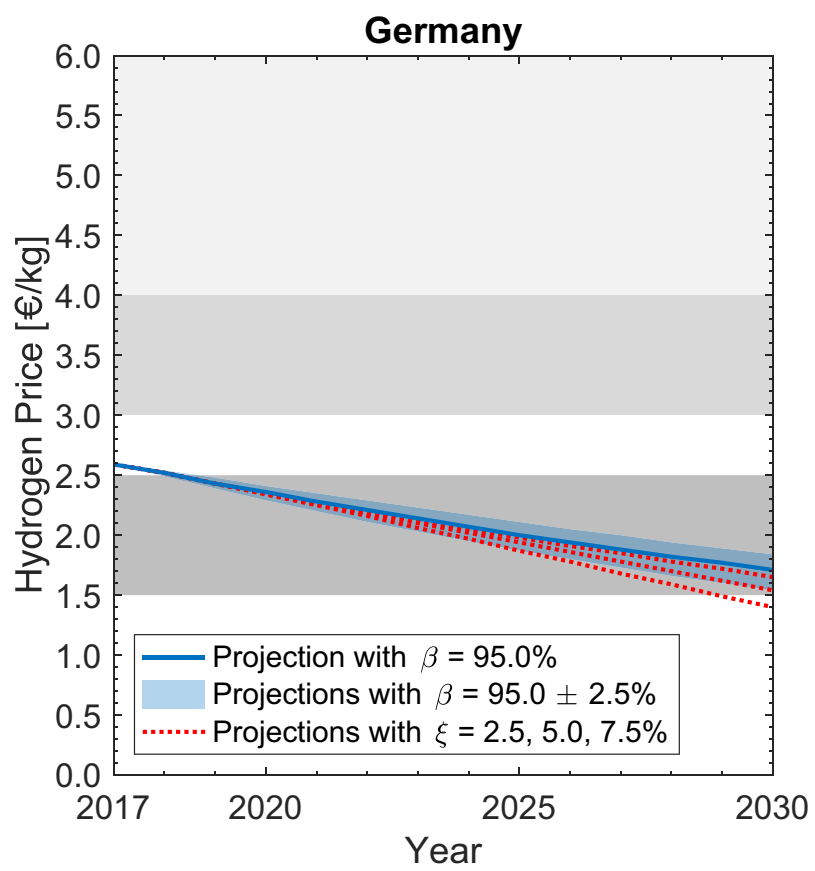

percentage at all other times so that $p^{s}$ remains unchanged. The dotted red lines represent the effect of $\xi$ values set equal to $2.5 \%, 5.0 \%$ and $7.5 \%$, respectively.

\section{Conclusion}

This study has examined the synergistic investment value of vertically integrated production systems. Synergies arise because of market imperfections for an intermediate input (electricity in our context) and because of operational volatility in the form of temporal fluctuations in output and prices. While vertically integrated systems will generally experience some synergistic benefit, we attribute a synergistic investment value only if a negative NPV for one or both of the stand-alone systems is more than outweighed by the synergistic effect. In the context of an energy system that combines renewable energy with hydrogen production, we derive necessary and sufficient conditions for the presence of the synergistic investment value. These conditions can be stated in terms of lifecycle unit costs and average prices adjusted for covariance terms that capture the extent to which price premia and output fluctuations are aligned across the hours of a typical year.

We rely on recent production price and cost data to assess the magnitude of synergistic effects in both Texas and Germany. Our empirical focus is on PtG facilities that can draw electricity either from the grid or internally from wind turbines. The policy support for renewable energy in Germany ensures that wind

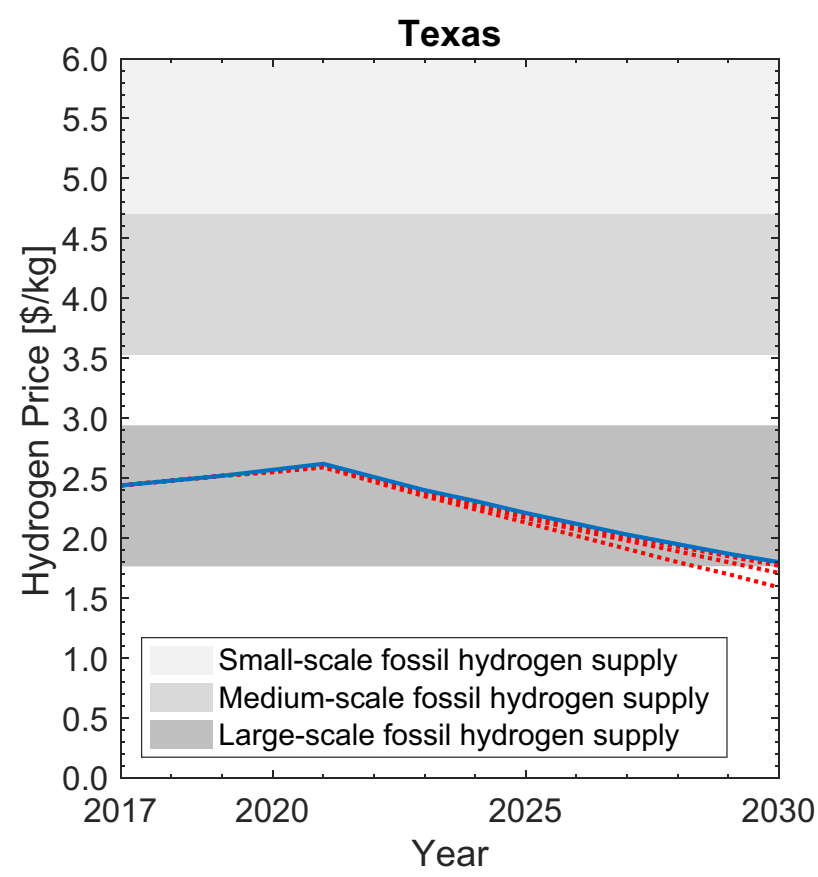

power is cost competitive on its own. We find that the emergence of a synergistic investment value in Germany hinges on the market price of hydrogen being above some break-even value which is currently below the price paid for medium-scale transactions, but above that obtained for industrial-scale transactions.

Owing to the low wholesale prices of electricity in Texas, we find that, wind energy on its own is currently not cost competitive despite the PTC available to renewable energy in the United States. Nevertheless we find that the synergies between the two subsystems are sufficiently strong in Texas so that a vertically integrated energy system can create value, despite the fact that PtG facilities will also not be viable on their own.

While our numerical analysis is based on the most recent available data, several factors suggest a trend towards a more favorable economics for vertically integrated systems in the future. We base our forecast based on the combination of projected reductions in system prices for both wind turbines and electrolyzers as well as a general trend towards more volatility in electricity prices.

Our paper suggests several promising avenues for future research. With regard to the modeling part, it would be instructive to add stochastic shocks to prices and output. Such shocks are likely to increase the call option value of capacity investments, but it remains an open question whether additional volatility in the form of random shocks will lead to synergistic investment values for a broader range of 
circumstances. We also note that our framework has viewed hydrogen as a final product. An alternative and promising avenue is to view hydrogen also as a form of electricity storage. Provided the electrolyzer can also run in the "reverse direction," hydrogen production coupled with reconversion to electricity may effectively compete with battery storage for electricity supply systems characterized by intermittent generation patterns.

\section{Acknowledgments}

We gratefully acknowledge financial support through the Deutsche Forschungsgemeinschaft (DFG, German Research Foundation) - Project-ID 403041268 - TRR 266. This research was also supported by the Hanns-Seidel-Stiftung with funds from the Federal Ministry of Education and Research of Germany. Helpful comments were provided by Stefanie Burgahn, Stephen Comello, Gunther Friedl, Christian Stoll, and colleagues at the TUM School of Management. Finally, we thank Florian Steffen for providing valuable assistance with our data collection.

\section{Appendix}

Proof of Lemma 1. We show that the claim in the statement applies to the four phases identified in Figure 2 of the main text. In Phase 1, both electricity prices exceed the contribution margin of hydrogen: $p^{b}(t) \geq p^{s}(t) \geq C V_{h} \geq 0$. The operator will idle the PtG facility and only sell renewable energy. The optimized contribution margin of the vertically integrated energy system will be equal to the contribution margin of the renewable energy source. To see this, note that:

$$
\begin{aligned}
p^{+}(t) & =\max \left\{\min \left\{p^{b}(t), C V_{h}\right\}, p^{s}(t)\right\} \\
& =\max \left\{C V_{h}, p^{s}(t)\right\}=p^{s}(t) .
\end{aligned}
$$

Furthermore:

$$
p^{b+}(t)=\max \left\{p^{b}(t), C V_{h}\right\}=p^{b}(t) .
$$

The optimized contribution margin of the vertically integrated energy system in Equation (9) thus reduces to:

$$
C M\left(t \mid k_{e}, k_{h}\right)=p^{s}(t) \cdot C F(t) \cdot k_{e} .
$$

In Phase 2, the buying price exceeds the contribution margin of hydrogen, which, in turn, exceeds the selling price: $p^{b}(t) \geq C V_{h}>p^{s}(t) \geq 0$. The facility will then convert the generated renewable energy without purchases of additional energy from the market. We find that:

$$
\begin{aligned}
p^{+}(t) & =\max \left\{\min \left\{p^{b}(t), C V_{h}\right\}, p^{s}(t)\right\} \\
& =\max \left\{C V_{h}, p^{s}(t)\right\}=C V_{h},
\end{aligned}
$$

and $p^{+}(t)-p^{s}(t)=C V_{h}-p^{s}(t)$. Since $p^{b}(t) \geq C V_{h}$, it follows that $p^{b+}(t)-p^{b}(t)=0$. Consistent with (11), the optimized contribution margin of the vertically integrated energy system reduces to the contribution margin of the renewable energy source plus the conversion premium of renewable energy:

$$
\begin{aligned}
C M\left(t \mid k_{e}, k_{h}\right)= & p^{s}(t) \cdot C F(t) \cdot k_{e}+\left[C V_{h}-p^{s}(t)\right] \\
& \cdot z\left(t \mid k_{e}, k_{h}\right) .
\end{aligned}
$$

In Phase 3, both electricity prices are less than the contribution margin of hydrogen and non-negative: $C V_{h}>p^{b}(t) \geq p^{s}(t) \geq 0$. The plant will convert the generated renewable energy and buy energy from the market to produce as much hydrogen as possible. Thus:

$$
\begin{aligned}
C M\left(t \mid k_{e}, k_{h}\right)= & p^{s}(t) \cdot C F(t) \cdot k_{e} \\
& +\left[C V_{h}-p^{s}(t)\right] \cdot z\left(t \mid k_{e}, k_{h}\right) \\
& +\left[C V_{h}(t)-p^{b}(t)\right] \cdot\left[k_{h}-z\left(t \mid k_{e}, k_{h}\right)\right] .
\end{aligned}
$$

Equivalently:

$$
\begin{aligned}
C M\left(t \mid k_{e}, k_{h}\right)=p^{s}( & t) \cdot C F(t) \cdot k_{e} \\
+ & {\left[p^{b}(t)-p^{s}(t)\right] \cdot z\left(t \mid k_{e}, k_{h}\right) } \\
& +\left[C V_{h}(t)-p^{b}(t)\right] \cdot k_{h} .
\end{aligned}
$$

In this scenario:

$$
\begin{aligned}
p^{+}(t) & =\max \left\{\min \left\{p^{b}(t), C V_{h}\right\}, p^{s}(t)\right\} \\
& =\max \left\{p^{b}(t), p^{s}(t)\right\}=p^{b}(t),
\end{aligned}
$$

and therefore $p^{+}(t)-p^{s}(t)=p^{b}(t)-p^{s}(t)$. Furthermore:

$$
p^{b+}(t)=\max \left\{p^{b}(t), C V_{h}\right\}=C V_{h},
$$

so that $p^{b+}(t)-p^{b}(t)=C V_{h}-p^{b}(t)$ and (A7) coincides with (9).

Finally, the buying price is negative in Phase 4. By assumption, $p^{b}(t) \leq 0=p^{s}(t)$. The plant operator will only buy energy from the market to convert it to hydrogen and refrain from selling renewable energy. We find that:

$$
\begin{aligned}
p^{+}(t) & =\max \left\{\min \left\{p^{b}(t), C V_{h}\right\}, p^{s}(t)\right\} \\
& =\max \left\{p^{b}(t), p^{s}(t)\right\}=p^{s}(t),
\end{aligned}
$$

and $p^{+}(t)-p^{s}(t)=0$. Furthermore:

$$
p^{b+}(t)=\max \left\{p^{b}(t), C V_{h}\right\}=C V_{h},
$$

and $p^{b+}(t)-p^{b}(t)=C V_{h}-p^{b}(t)$. The expression in Equation (11) therefore reduces to the contribution margin of a stand-alone PtG plant running on grid electricity only:

$$
C M\left(t \mid k_{e}, k_{h}\right)=\left[C V_{h}(t)-p^{b}(t)\right] \cdot k_{h} .
$$


Proof of Proposition 1. The NPV of a vertically integrated energy system is given by the present value of future operating cash flows less the initial capacity investment:

$N P V\left(k_{e}, k_{h}\right)=\sum_{i=1}^{T} C F L_{i}\left(k_{e}, k_{h}\right) \cdot \gamma^{i}-\left(k_{e} \cdot S P_{e}+k_{h} \cdot S P_{h}\right)$,

with $C F L_{i}\left(k_{e}, k_{h}\right)$ as the after-tax operating cash flow in year $i$. It is given by the difference between the pre-tax cash flow in year $i, C F L_{i}^{o}\left(k_{e}, k_{h}\right)$, and current corporate income taxes, given by the tax rate, $\alpha$, applied to the taxable income, $I_{i}\left(k_{e}, k_{h}\right)$ :

$$
C F L_{i}\left(k_{e}, k_{h}\right)=C F L_{i}^{o}\left(k_{e}, k_{h}\right)-\alpha \cdot I_{i}\left(k_{e}, k_{h}\right) .
$$

The pre-tax operating cash flow in year $i$ comprises the optimized contribution margin of a vertically integrated energy system less the fixed operating costs:

$$
C F L_{i}^{o}\left(k_{e}, k_{h}\right)=x^{i-1} \int_{0}^{m} C M\left(t \mid k_{e}, k_{h}\right) d t-\left(k_{e} \cdot F_{e i}+k_{h} \cdot F_{h i}\right),
$$

with $x<1$ denoting the degradation factor, that is, the percentage by which the capacity declines in each subsequent year. The firm's taxable income in year $i$ is then given by the pre-tax cash flow less depreciation, with $d_{i}$ denoting the allowable depreciation percentage in year $i$. For simplicity, we assume that the same depreciation schedule applies to all components of the vertically integrated energy system:

$$
I_{i}\left(k_{e}, k_{h}\right)=\operatorname{CFL}_{i}^{o}\left(k_{e}, k_{h}\right)-\left(k_{e} \cdot S P_{e}+k_{h} \cdot S P_{h}\right) \cdot d_{i} .
$$

Combining the expressions in Equation (A14) (A15), and (A16), the NPV becomes:

$$
\begin{aligned}
N P V\left(k_{e}, k_{h}\right) & =(1-\alpha) \cdot\left[\sum _ { i = 1 } ^ { T } \gamma ^ { i } \cdot \left(x^{i-1} \int_{0}^{m} C M\left(t \mid k_{e}, k_{h}\right) d t\right.\right. \\
& \left.\left.-\left(k_{e} \cdot F_{e i}+k_{h} \cdot F_{h i}\right)\right)\right] \\
& -\left(1-\alpha \sum_{i=1}^{T} d_{i} \cdot \gamma^{i}\right) \cdot\left(k_{e} \cdot S P_{e}+k_{h} \cdot S P_{h}\right) .
\end{aligned}
$$

Since the tax factor was defined as:

$$
\Delta=\frac{1-\alpha \cdot \sum_{i=1}^{T} d_{i} \cdot \gamma^{i}}{1-\alpha},
$$

the expression for the NPV reduces to:

$$
\begin{aligned}
N P V\left(k_{e}, k_{h}\right) & =(1-\alpha) \cdot\left[\sum _ { i = 1 } ^ { T } \gamma ^ { i } \cdot \left(x^{i-1} \int_{0}^{m} C M\left(t \mid k_{e}, k_{h}\right) d t\right.\right. \\
& \left.\left.-\left(k_{e} \cdot F_{e i}+k_{h} \cdot F_{h i}\right)\right)-\Delta \cdot\left(k_{e} \cdot S P_{e}+k_{h} \cdot S P_{h}\right)\right] .
\end{aligned}
$$

It will be convenient to pull out the "levelization" factor $L \equiv m \cdot \sum_{i=1}^{T} x^{i-1} \cdot \gamma^{i}$ :

$$
\begin{aligned}
N P V\left(k_{e}, k_{h}\right) & =(1-\alpha) \cdot L \cdot\left[\frac{1}{m} \int_{0}^{m} C M\left(t \mid k_{e}, k_{h}\right) d t\right. \\
& -\frac{\sum_{i=1}^{T} \gamma^{i} \cdot\left(k_{e} \cdot F_{e i}+k_{h} \cdot F_{h i}\right)}{m \cdot \sum_{i=1}^{T} x^{i-1} \cdot \gamma^{i}} \\
& \left.-\Delta \cdot \frac{k_{e} \cdot S P_{e}+k_{h} \cdot S P_{h}}{m \cdot \sum_{i=1}^{T} x^{i-1} \cdot \gamma^{i}}\right] .
\end{aligned}
$$

(A20)

The main text introduced the levelized cost of electricity of the renewable energy source as $L C O E=f_{e}+\Delta \cdot c_{e}$ (assuming a zero variable cost for generating renewable electricity), and $L F C H=f_{h}+\Delta \cdot c_{h}$. Here, $f_{e}$ and $f_{h}$ refer to the timeaveraged operating fixed costs and $c_{e}$ and $c_{h}$ to the unit costs of capacity of the two subsystems. We thus obtain:

$$
\begin{aligned}
N P V\left(k_{e}, k_{h}\right)= & (1-\alpha) \cdot L \cdot\left[\frac{1}{m} \int_{0}^{m} C M\left(t \mid k_{e}, k_{h}\right) d t\right. \\
& \left.-C F \cdot k_{e} \cdot L C O E-k_{h} \cdot L F C H\right] .
\end{aligned}
$$

Lemma 1 allows us to substitute the following expression for the optimized contribution margin:

$$
\begin{aligned}
N P V\left(k_{e}, k_{h}\right) & =(1-\alpha) \cdot L \cdot\left[\frac { 1 } { m } \left(k_{e} \int_{0}^{m} p^{s}(t) \cdot C F(t) d t\right.\right. \\
& +\int_{0}^{m}\left[p^{+}(t)-p^{s}(t)\right] \cdot z\left(t \mid k_{e}, k_{h}\right) d t \\
& \left.+k_{h} \int_{0}^{m}\left(p^{b+}(t)-p^{b}(t)\right) d t\right) \\
& \left.-C F \cdot k_{e} \cdot \text { LCOE }-k_{h} \cdot L F C H\right] .
\end{aligned}
$$


The final step accounts for the temporal covariations in prices and capacity factors. We recall from the main text that $\varepsilon(t)$ denotes the multiplicative deviation factor of $C F(t)$ from the yearly average $C F$ and $\mu(t)$ as the multiplicative deviation factor of $p^{s}(t)$ from the yearly average $p^{s}$ so that:

$$
\epsilon(t)=\frac{C F(t)}{C F} \text { and } \mu(t)=\frac{p^{\varsigma}(t)}{p^{s}} .
$$

The average capacity factor is given by $C F=\frac{1}{m} \int_{0}^{m} C F(t) d t$ and the average selling price is given by $p^{s}=\frac{1}{m} \int_{0}^{m} p^{s}(t) d t$. The covariation between output and the price can then be captured by the covariation coefficient:

$$
\Gamma^{s}=\frac{1}{m} \int_{0}^{m} \epsilon(t) \cdot \mu(t) d t
$$

We further recall that, by definition, $\mu^{+}(t) \cdot\left(p^{+}-p^{s}\right)=p^{+}(t)-p^{s}(t)$ and

$$
z\left(k_{e}, k_{h}\right) \equiv \frac{1}{m} \int_{0}^{m} z\left(t \mid k_{e}, k_{h}\right) \cdot \mu^{+}(t) d t .
$$

Taken together, the expression for the NPV simplifies to:

$$
\begin{aligned}
N P V\left(k_{e}, k_{h}\right)= & (1-\alpha) \cdot L \cdot\left[\left(\Gamma^{s} \cdot p^{s}-L C O E\right) \cdot C F \cdot k_{e}\right. \\
& +\left(p^{b+}-p^{b}-L F C H\right) \cdot k_{h} \\
& \left.+\left(p^{+}-p^{s}\right) \cdot z\left(k_{e}, k_{h}\right)\right] .
\end{aligned}
$$

Proof of Corollary to Proposition 1. If both standalone systems are cost competitive, the vertically integrated energy system has synergistic investment value whenever:

$$
p^{+}-p^{s}>0 \text {. }
$$

It follows directly from the definition of $p^{+}(t)$ that:

$$
p^{+}(t)=\max \left\{\min \left\{C V_{h}, p^{b}(t)\right\}, p^{s}(t)\right\} \geq p^{s}(t),
$$

and that (A27) is fulfilled if and only if it holds that:

$$
\min \left\{C V_{h}, p^{b}(t)\right\}>p^{s}(t) \text { for some } t .
$$

Proof of Proposition 2. (i) For sufficiency, we show that at $k_{e}=1$ :

$$
\left.\frac{\partial}{\partial k_{h}} N P V\left(k_{e}=1, k_{h}\right)\right|_{k_{h}=0}>0,
$$

if the inequality in Equation (28) holds.

$$
\begin{aligned}
\left.\frac{\partial}{\partial k_{h}} N P V\left(1, k_{h}\right)\right|_{k_{h}=0}= & \left(p^{+}-p^{s}\right) \cdot \frac{\partial}{\partial k_{h}} z(1,0) \\
& +\left(p^{b+}-p^{b}\right)-L F C H \\
= & \left(p^{+}-p^{s}\right)+\left(p^{b+}-p^{b}\right)-L F C H>0 .
\end{aligned}
$$

For necessity, suppose the condition in Equation (28) is not met, yet the vertically integrated energy system exhibits synergistic investment value and thus $N P V\left(1, k_{h}\right) \geq N P V(1,0)$ for some $k_{h}$. We obtain:

$$
\begin{gathered}
N P V\left(1, k_{h}\right)-N P V(1,0)=\int_{0}^{k_{h}} \frac{\partial}{\partial k_{h}} N P V(1, u) d u \\
=\int_{0}^{k_{h}}\left[\left(p^{+}-p^{s}\right) \cdot \frac{\partial}{\partial k_{h}} z(1, u)+\left(p^{b+}-p^{b}\right)-L F C H\right] d u \\
\leq \int_{0}^{k_{h}}\left[\left(p^{+}-p^{s}\right)+\left(p^{b+}-p^{b}\right)-L F C H\right] d u \\
=k_{h} \cdot\left[\left(p^{+}-p^{s}\right)+\left(p^{b+}-p^{b}\right)-L F C H\right] \\
<0,
\end{gathered}
$$

a contradiction.

If there is synergistic investment value, the firstorder condition for the optimal $k_{h}^{*}(1)$ is:

$$
\begin{aligned}
\frac{\partial}{\partial k_{h}} N P V\left(1, k_{h}^{*}(1)\right)= & \frac{\partial}{\partial k_{h}} z\left(1, k_{h}^{*}(1)\right) \cdot\left(p^{+}-p^{s}\right) \\
& +\left(p^{b+}-p^{b}\right)-L F C H=0 .
\end{aligned}
$$

The value of $k_{h}^{*}(1)$ is unique because $\frac{\partial}{\partial k_{h}} z(1, \cdot)$ is monotone decreasing in $k_{h}$. Furthermore, $N P V(1, \cdot)$ is increasing in $k_{h}$ up to $k_{h}^{*}(1)$ and decreasing thereafter.

The proof of part (ii) is entirely symmetric.

Proof of Proposition 3. If neither stand-alone system is cost competitive, the vertically integrated energy system has synergistic investment value whenever:

$$
N P V\left(1, k_{h}^{*}(1)\right) \geq 0
$$


It follows directly from the characterization of the $N P V\left(k_{e}, k_{h}\right)$ in Proposition 2 that $N P V\left(1, k_{h}^{*}(1)\right)$ is proportional to:

$$
\begin{array}{r}
\left(p^{+}-p^{s}\right) \cdot z\left(1, k_{h}^{*}(1)\right)+\left(p^{b+}-p^{b}-L F C H\right) \cdot k_{h}^{*}(1) \\
+\left(\Gamma^{s} \cdot p^{s}-L C O E\right) \cdot C F,
\end{array}
$$

thus establishing the claim.

Proof of Corollary to Proposition 3. Suppose the vertically integrated system has synergistic investment value, that is, $N P V\left(1, k_{h}^{*}(1)\right)>0$. Proposition 2 established that $k_{h}^{*}(1)>0$ if only if $p^{+}-p^{s}+p^{b+}-p^{b}-L F C H>0$. Now suppose that, contrary to the claim, $p^{+}-p^{s}+p^{b+}-p^{b}-L F C H$ $+\left(\Gamma^{s} \cdot p^{s}-L C O E\right) \cdot C F<0$. It would then follow that:

$$
\begin{aligned}
& N P V\left(1, k_{h}^{*}(1)\right)=(1-\alpha) \cdot L\left[\left(p^{+}-p^{s}\right) \cdot z\left(1, k_{h}^{*}(1)\right)\right. \\
& +\left(p^{b+}-p^{b}-L F C H\right) \cdot k_{h}^{*}(1) \\
& \left.+\left(\Gamma^{s} \cdot p^{s}-L C O E\right) \cdot C F\right] \\
& \leq(1-\alpha) \cdot L\left[\left(p^{+}-p^{s}\right) \cdot k_{h}^{*}(1)+\left(p^{b+}-p^{b}-L F C H\right) \cdot k_{h}^{*}(1)\right. \\
& \left.+\left(\Gamma^{s} \cdot p^{s}-L C O E\right) \cdot C F\right]
\end{aligned}
$$$$
\leq(1-\alpha) \cdot L\left[\left(p^{+}-p^{s}\right)+p^{b+}-p^{b}-L F C H\right.
$$$$
\left.+\left(\Gamma^{s} \cdot p^{s}-L C O E\right) \cdot C F\right]
$$

$$
<0 \text {. }
$$

The first inequality follows from the observation

\begin{tabular}{|c|c|c|c|}
\hline Price & Unit & Value & Source \\
\hline Trading cost & $€ \varnothing / \mathrm{kWh}$ & 1.0000 & Industry experts \\
\hline Transmission charge & $€ \varnothing / \mathrm{kWh}$ & 0.0000 & EnWG (2005) §118 (6)) \\
\hline Concession charge & $€ \varnothing / \mathrm{kWh}$ & 0.1100 & KAV (1992) §2 (3) 1.) \\
\hline EEG-Levy & $€ \varnothing / \mathrm{kWh}$ & 0.1000 & EEG (2014) §64 with A.4) \\
\hline CHP markup & $€ \notin / \mathrm{kWh}$ & 0.0830 & KWKG (2016) $\S 9(7))$ \\
\hline$\S 19$ StromNEV levy & $€ \notin / k W h$ & 0.0510 & StromNEV (2016) §19 (2)) \\
\hline Offshore liability levy & $€ \notin / \mathrm{kWh}$ & 0.0270 & EnWG (2005) §17f) \\
\hline $\begin{array}{l}\text { Levy for interruptible } \\
\text { loads }\end{array}$ & $€ \notin / \mathrm{kWh}$ & 0.0000 & AbLaV (2012) §18) \\
\hline Electricity tax & $€ \varnothing / k W h$ & 0.0000 & StromStG (2016) $\S 9 a(1) 1)$. \\
\hline $\begin{array}{l}\text { Total industry } \\
\text { price markup }\end{array}$ & $€ \varnothing / \mathrm{kWh}$ & 1.3710 & \\
\hline
\end{tabular}
that, by definition, $z\left(1, k_{h}^{*}(1)\right) \leq k_{h}^{*}(1)$, while the second inequality relies on $k_{h}^{*}(1) \leq 1$ due to the fact that $\lim _{k_{h} \rightarrow 1} \frac{\partial}{\partial k_{h}} z\left(1, k_{h}\right)=0$.

\section{Structures of Electricity Buying Prices}

In Germany, the PtG facility can buy electricity from the wholesale market subject to a markup of taxes, fees and levies:
In Texas, we use the industrial rate "Primary < 3MW" by Austin Energy (2014) without time-of-use prices since they have been suspended for new customers. Water electrolysis is exempted from state and local sales tax (Texas Tax Code 2016, \$2.151.317 (a) (6)). Thus, the rate is:

\begin{tabular}{llll}
\hline Price & Unit & Summer & Winter \\
\hline Customer charge & $\$ /$ month & 236.00 & 236.00 \\
Electric delivery & $\$ / k W$ & 2.36 & 2.36 \\
Demand charge & $\$ / k W$ & 9.44 & 8.44 \\
Regulatory charge & $\$ / k W$ & 6.75 & 6.75 \\
Energy charge & $\$ \notin / k W h$ & 1.1920 & 0.7200 \\
Power supply adjustment & $\$ \notin / k W h$ & 2.7200 & \\
CBC: Customer assistance program & $\$ \notin / k W h$ & 0.0650 & \\
CBC: Energy efficiency services & $\$ \notin / k W h$ & 0.2520 & \\
Gross Receipt Tax & $\%$ & 1.997 & \\
State and local Tax & $\%$ & 0 & \\
\hline
\end{tabular}

A PtG facility can provide frequency control services. In Texas, this is referred to as "regulation down," while it is referred to as "negative Sekundärregelleistung" in Germany (ERCOT 2017, Regelleistung.net. 2017). In both jurisdictions, frequency control is compensated with a capacity price per $\mathrm{kW}$ that the facility is in standby. In Germany, the facility is also paid a price per kWh of energy absorbed from the grid. Since both types of payment reflect negative buying prices, we assume the facility always offers this service. The buying price for open market energy can then be expressed as the weighted average of the energy price for frequency control and the market price:

$$
p^{b}(t)=\phi(t) \cdot p^{c}(t)+(1-\phi(t)) \cdot p^{m}(t),
$$

where $p^{c}(t)$ denotes the price for calling energy per $\mathrm{kWh}, \phi(t)$ the share of called capacity in hour $t$, and $p^{m}(t)$ the price for market energy per $\mathrm{kWh}$. The capacity price, on the other hand, adds to the conversion premium of hydrogen. Since the price is paid per $\mathrm{kW}$, we divide it by the hours of standby to receive a price per $\mathrm{kWh}$. With $p^{s b}$ denoting the time-averaged standby capacity price, the NPV of stand-alone PtG becomes:

$$
N P V\left(k_{h}\right)=(1-\alpha) \cdot L \cdot\left(p^{b+}-p^{b}-p^{s b}-L F C H\right) \cdot k_{h} .
$$

Offering frequency control requires the $\mathrm{PtG}$ facility to absorb electricity when called, which effectively reduces the capacity to convert renewable energy. Thus, $z\left(t \mid k_{e}, k_{h}\right)$ becomes:

$$
z\left(t \mid k_{e}, k_{h}\right) \equiv \min \left\{C F(t) \cdot k_{e},(1-\phi(t)) \cdot k_{h}\right\}
$$


List of Valuation Parameters

\begin{tabular}{|c|c|c|c|}
\hline & Germany & Texas & Source \\
\hline \multicolumn{4}{|l|}{ General } \\
\hline Economic lifetime, $T$ & 30 years & 30 years & Michalski et al. (2017) \\
\hline Corporate income tax rate, $\alpha$ & $35.00 \%$ & $21.00 \%$ & German and U.S. Tax Code \\
\hline Degradation rate, $x_{i}$ & $0.80 \%$ & $0.80 \%$ & Deutsche WindGuard (2013), Fraunhofer ISE (2013) \\
\hline Depreciation rate, $d_{i}$ & $6.25 \%$ (16y linear) & $100 \%$ Bonus & Bundesfinanzhof (2011), U.S. Congress (2017) \\
\hline Cost of capital (WACC), $r$ & $4.00 \%$ & $6.00 \%$ & Fraunhofer ISI (2016), Moné et al. (2015) \\
\hline Subsidy lifetime & 20 years & 10 years & EEG (2017), U.S. Department of Energy (2016) \\
\hline \multicolumn{4}{|l|}{ Wind energy } \\
\hline Capacity factor, CF & $30.33 \%$ & $44.39 \%$ & Own data, Wallasch et al. (2016), ABB (2017) \\
\hline Variable operating cost, $w_{e}$ & $0.00 € / \mathrm{kWh}$ & $0.00 \$ / \mathrm{kWh}$ & Negligible cost, ABB (2017) \\
\hline Fixed operating cost, $F_{e}$ & $38.00 € / \mathrm{kW}$ & $21.70 \$ / \mathrm{kW}$ & Wallasch et al. (2016), ABB (2017) \\
\hline Acquisition cost, $S P_{e}$ & $1,180 € / \mathrm{kW}$ & $1,566 \$ / \mathrm{kW}$ & Fraunhofer IWES (2017), ABB (2017) \\
\hline \multicolumn{4}{|l|}{ Power-to-Gas } \\
\hline Conversion rate, $\eta$ & 0.019 kg/kWh & $0.019 \mathrm{~kg} / \mathrm{kWh}$ & Bertuccioli et al. (2014) \\
\hline Variable operating cost, $w_{h}$ & $0.10 € / \mathrm{kg}$ & $0.08 \$ / \mathrm{kg}$ & Estimation of water cost. ${ }^{*}$ \\
\hline Fixed operating cost, $F_{h}$ & $45.00 € / \mathrm{kW}$ & $39.50 \$ / \mathrm{kW}$ & Glenk and Reichelstein (2019) \\
\hline Acquisition cost, $S P_{h}$ & $2,074 € / \mathrm{kW}$ & $1,822 \$ / \mathrm{kW}$ & Glenk and Reichelstein (2019) \\
\hline \multicolumn{4}{|l|}{ Prices } \\
\hline Selling price of electricity, $p^{s}$ & $3.42 € \notin / \mathrm{kWh}$ & $2.45 \$ \notin / \mathrm{kWh}$ & http://www.eex.com, http://www.ercot.com \\
\hline Buying price of electricity, $p^{b}$ & $3.93 € \phi / k W h$ & $5.39 \$ \notin / \mathrm{kWh}$ & See below \\
\hline
\end{tabular}

${ }^{*}$ Conversion to \$ with average exchange rate of 2015 (1.1104 \$/€, see European Central Bank) and U.S. state index (0.7910, Comello et al. (2018)).

\section{Notes}

${ }^{1}$ Hydrogen can then be used in a wide range of applications including fuel for transportation, feedstock in chemical and processing industries, and energy storage for power generation.

${ }^{2}$ The costs and benefits of vertical integration have long been central issues in the theory of the firm (Williamson 1975, 1985). Much of the literature in economics has approached these issues from an incentive and management control perspective; see, for instance, Grossman and Hart (1986), Melumad et al. (1995), and Gilbert and Riordan (1995). Our approach in this study is in line with recent perspectives in the operations literature, e.g., Kazaz (2004), van Mieghem (2003), and $\mathrm{Hu}$ et al. (2015). In these studies, the benefits of vertically integrated production systems generally stem from operational gains, while costs arise from the need for additional upfront investments in productive capacity.

${ }^{3}$ See, for instance, Zhou et al. (2016), Broneske and Wozabal (2016), Wozabal et al. (2016).

${ }^{4}$ Recent work on vertical integration has been confined to settings where one subsystem can be added to the other, yet the added subsystem can not operate stand-alone (Boyabatli et al. 2017, Dong et al. 2014, Kazaz 2004).

${ }^{5}$ Our results are also related to the real option literature which examines whether the value of a flexible system exceeds that of a rigid system sufficiently so as to justify investment in the flexible system, e.g., Kogut and Kulatilaka (1994), van Mieghem (1998), Trigeorgis (1993). output is assumed to be fully dispatchable in these studies. By including exogenous output fluctuations, our study is partly in the spirit of the hedging literature. McKinnon (1967) and Rolfo (1980) examine when farmers can effectively hedge by selling a share of their crops on the futures market instead of selling everything on the spot market.
The analogy with our setting is that, instead of hedging with a price future, farmers could also invest in optimally sized equipment that turns the crops into products with a stable market price.

${ }^{6}$ For technical reasons, we assume that $C F(t)>0$ and that each value in the range of the function $C F(\cdot)$ is assumed at most finitely many times. These assumptions appear descriptive for wind turbines, the setting we examine in section 4 below.

${ }^{7}$ Proofs are shown in the Appendix.

${ }^{8}$ As shown in Reichelstein and Rohlfing-Bastian (2015), this cost measure is also the relevant unit cost for optimal capacity investment decisions in the presence of future random shocks to demand.

${ }^{9}$ This formulation entails the implicit assumption that maintenance of the PtG facility can be performed at times when the system idle.

${ }^{10}$ Our argument here assumes implicitly that the functions $p^{b}(\cdot)$ and $p^{s}(\cdot)$ are continuous functions.

${ }^{11}$ To see this, we note that the concavity of the function $N P V(\cdot, \cdot)$ in Equation (24) hinges on $z\left(k_{e}, k_{h}\right)$ being concave in $\left(k_{e}, k_{h}\right)$. For any given $t$, the concavity argument for $\min \left\{C F(t) \cdot k_{e}, k_{h}\right\} \cdot \mu^{+}(t)$ follows directly from the observation that the function $\min \{A, B\}$ is concave in $(A, B)$.

${ }^{12}$ In its current form, this premium is only granted for wind energy fed into the grid. Our subsequent calculations assume that this premium would also be granted for renewable electricity that is converted to hydrogen, that is, the renewable energy is effectively stored.

${ }^{13}$ The profit margin in Germany is given by $\Gamma^{s} \cdot p^{s}+P P-L C O E$ and in Texas by $\Gamma^{s} \cdot p^{s}-L C O E$ since the LCOE in Texas includes the PTC reduction.

${ }^{14}$ Our calculations are based on a polymer electrolyte membrane (PEM) electrolyzer, which is the most flexible electrolyzer technology in terms of ramping delays (Buttler and Spliethoff 2018). 
${ }^{15}$ We note in passing that the results reported in Figure 5 are based on our figures reported above where the medium-scale supply price of hydrogen in Texas was benchmarked at $4.00 \$ / \mathrm{kg}(3.50 € / \mathrm{kg}$ in Germany), while the large-scale supply prices were set at $2.50 \$ / \mathrm{kg}$ in Texas and $2.00 € / \mathrm{kg}$ in Germany.

${ }^{16}$ This is the approach in Figure 5. We note that the question of a synergistic investment value cannot be posed in such a context since, by construction, hydrogen can only be produced by the vertically integrated facility.

\section{References}

ABB. 2017. Velocity suite - market intelligence services.

AbLaV. 2012. Verordnung über vereinbarungen zu abschaltbaren lasten.

Ainscough, C., D. Peterson, E. Miller. 2014. Hydrogen production cost from PEM electrolysis. Technical report.

Austin Energy. 2014.City of Austin utility rates and fees schedule. Technical report.

Bertuccioli, L., A. Chan, D. Hart, F. Lehner, B. Madden, E. Standen. 2014. Study on development of water electrolysis in the EU. Technical report, Fuel Cells and Hydrogen Joint Undertaking.

Bloomberg. 2017. Big Energy Backs Hydrogen Power Storage. Available at https://tinyurl.com/y8a3efja (accessed date September 18, 2019).

Boyabatli, O., J. Nguyen, T. Wang. 2017. Capacity management in agricultural commodity processing and application in the palm industry. Manuf. Serv. Oper. Manag. 19(4): 551-567. ISSN 1523-4614.

Broneske G., D. Wozabal. 2016. How do contract parameters influence the economics of vehicle-to-grid? Manuf. Serv. Oper. Manag. 19(1): 1-34. ISSN 1523-4614.

Bundesfinanzhof. 2011. BFH-Urteil 14.04.2011 IV R 52/10. Bundesfinanzhof.

Buttler A., H. Spliethoff. 2018. Current status of water electrolysis for energy storage, grid balancing and sector coupling via power-to-gas and power-to-liquids: A review. Renew. Sustain. Energy Rev. 82: 2440-2454. ISSN 18790690.

Comello, S., G. Glenk, S. Reichelstein. 2018. Levelized cost of electricity calculator. Available at https://tinyurl.com/ yb5aac92 (accessed date July 5, 2019).

Davis, S. J., N. S. Lewis, M. Shaner, S. Aggarwal, D. Arent, I. L. Azevedo, S. M. Benson, T. Bradley, J. Brouwer, Y.-M. Chiang, C. T. M. Clack, A. Cohen, S. Doig, J. Edmonds, P. Fennell, C. B. Field, B. Hannegan, B.-M. Hodge, M. I. Hoffert, E. Ingersoll, P. Jaramillo, K. S. Lackner, K. J. Mach, M. Mastrandrea, J. Ogden, F. Peterson, D. L. Sanchez, D. Sperling, J. Stagner, J. E. Trancik, C.-J. Yang, K. Caldeira. 2018. Net-zero emissions energy systems. Science 360(6396): 9793. ISSN 0036-8075.

Deutsche WindGuard. 2013. Kostensituation der windenergie an land in deutschland update. Technical report.

Dong, L., P. Kouvelis, X. Wu. 2014. The value of operational flexibility in the presence of input and output price uncertainties with oil refining applications. Management Sci. 60(12): 29082926. ISSN 0025-1909.

EEG. 2014. Gesetz für den ausbau erneuerbarer energien.

EEG. 2017. Gesetz für den ausbau erneuerbarer energien.

EnWG. 2005. Gesetz über die elektrizitäts- und gasversorgung.

ERCOT. 2017. Electric reliability Council of Texas.

Farhat K., S. Reichelstein. 2016. Economic value of flexible hydrogen-based polygeneration energy systems. Appl. Energy 164: 857-870. ISSN 0306-2619.
Felgenhauer M., T. Hamacher. 2015. State-of-the-art of commercial electrolyzers and on-site hydrogen generation for logistic vehicles in South Carolina. Int. J. Hydrogen Energy 40(5): 20842090. ISSN 03603199.

Fraunhofer ISE. 2013. Stromgestehungskosten erneuerbare energien. Technical report.

Fraunhofer ISI. 2016. The impact of risks in renewable energy investments and the role of smart policies. Technical report.

Fraunhofer IWES. 2017. Windenergie report deutschland 2016. Technical report.

Gilbert R. J., M. H. Riordan. 1995. Regulating complementary products: A comparative institutional analysis. Rand. J. Econ. 26(2): 243-256.

Glenk G., and S. Reichelstein. 2019. Economics of converting renewable power to hydrogen. Nat. Energy 4: 216-222. ISSN 2058-7546.

Grossman S. J., O. D. Hart. 1986. The costs and benefits of ownership: A theory of vertical and lateral integration. J. Polit. Econ. 94(4): 691-719. ISSN 0022-3808.

GTM. 2018. Australia seeks hydrogen to soak up excess renewable energy production.

Hekimoglu, M. H., B. Kazaz, S. Webster. 2017. Wine analytics: Fine wine pricing and selection under weather and market uncertainty. Manuf. Serv. Oper. Manag. 19(2): 202-215. ISSN 1523-4614.

Hu, S., G. C. Souza. M. E. Ferguson. W. Wang

Islegen, Ö., S. Reichelstein. 2011. Carbon capture by fossil fuel power plants: An economic analysis. Management Sci. 57): 2139. ISSN 0025-1909.

ITM Power. 2018. World's largest hydrogen electrolysis in Shell's Rhineland Refinery.

KAV. 1992. Verordnung über konzessionsabgaben für strom und gas.

Kazaz, B. 2004. Production planning under yield and demand uncertainty with yield-dependent cost and price. Manuf. Serv. Oper. Manag. 6(3): 209-224. ISSN 1523-4614.

Ketterer, J. C. 2014. The impact of wind power generation on the electricity price in Germany. Energy Econ. 44: 270-280. ISSN 01409883.

Kogut, B., N. Kulatilaka. 1994. Operating flexibility, global manufacturing, and the option value of a multinational network. Management Sci. 40(1): 123-139. ISSN 0025-1909.

Kouvelis, P., D. Turcic, W. Zhao. 2018. Supply chain contracting in environments with volatile input prices and frictions. Manuf. Serv. Oper. Manag. 20(1): 130-146.

KWKG. 2016. Gesetz für die erhaltung, die modernisierung und den ausbau der kraft-Wärme-kopplung.

McKinnon, R. I. 1967. Futures markets, buffer stocks, and income stability for primary producers. J. Polit. Econ. 75(6): 844-861. ISSN 00223808.

Melumad, N. D., D. Mookherjee, S. Reichelstein. 1995. Hierarchical decentralization of incentive contracts. Rand J. Econ. 26(4): 654-672. ISSN 07416261.

Michalski, J., U. Bünger, F. Crotogino, S. Donadei, G. S. Schneider, T. Pregger, K. K. Cao, D. Heide. 2017. Hydrogen generation by electrolysis and storage in salt caverns: Potentials, economics and systems aspects with regard to the German energy transition. Int. J. Hydrogen Energy 42(19): 13427-13443. ISSN 03603199.

van Mieghem, J. A. 1998. Investment strategies for flexible resources. Management Sci. 44(8): 1071-1078.

van Mieghem, J. A. 2003. Capacity management, investment, and hedging: Review and recent developments. Manuf. Serv. Oper. Manag. 5(4): 269-302. ISSN 1523-4614. 
Moné, C., T. Stehly, B. Maples, E. Settle. 2015. 2014 cost of wind energy review. Technical report February.

Paraschiv, F., D. Erni, R. Pietsch. 2014. The impact of renewable energies on EEX day-ahead electricity prices. Energy Pol. 73: 196-210. ISSN 03014215.

Regelleistung.net. 2017. Internetplattform zur Vergabe von Regelleistung.

S. Reichelstein and A. Rohlfing-Bastian. 2015. Levelized product cost: Concept and decision relevance. Account. Rev. 90(4): 1653-1682. ISSN 0001-4826.

Reichelstein S., A. Sahoo. 2015. Time of day pricing and the levelized cost of intermittent power generation. Energy Econ. 48: 97-108. ISSN 0140-9883.

Rolfo, J. 1980. Optimal hedging under price and quantity uncertainty: The case of a cocoa producer. J. Polit. Econ. 88(1): 100116. ISSN 0022-3808.

StromNEV. 2016. Verordnung über die Entgelte für den Zugang zu Elektrizitätsversorgungsnetzen.

StromStG. 2016. Stromsteuergesetz.

Texas Tax Code. 2016. Gas and electricity.

Trigeorgis, L. 1993. The nature of option interactions and the valuation of investments with multiple real options. J. Financ. Quant. Anal. 28(1): 1-20. ISSN 00221090, 17566916.

U.S. Congress. 2017. H.R.1: An Act to provide for reconciliation pursuant to titles II and V of the concurrent resolution on the budget for fiscal year 2018 .
U.S. Department of Energy. 2016. Renewable electricity production tax credit(PTC).

de Véricourt F., D. Gromb. 2018. Financing capacity investment under demand uncertainty: An optimal contracting approach. Manuf. Serv. Oper. Manag. 20(1): 85-96.

Voestalpine. 2018. voestalpine und ihre Partner erhalten grünes Licht für den Bau der weltweit größten industriellen Wasserstoffpilotanlage in Linz.

Wallasch, A. -K., S. Lüers, K. Rehfeldt. 2016. Weiterbetrieb von Windenergie-Anlagen nach 2020. Technical report.

Williamson, O. E. 1975. Markets and Hierarchies. The Free Press, New York, NY.

Williamson, O. E. 1985. The Economic Institutions of Capitalism. The Free Press, New York, NY.

Wiser, R., K. Jenni, J. Seel, E. Baker, M. Hand, E. Lantz, A. Smith. 2016. Expert elicitation survey on future wind energy costs. Nat. Energy 1(10): 16135. ISSN 20587546.

Woo, C. K., I. Horowitz, J. Moore, A. Pacheco. 2011. The impact of wind generation on the electricity spot-market price level and variance: The Texas experience. Energy Pol. 39(7): 39393944. ISSN 03014215.

Wozabal, D., C. Graf, D. Hirschmann. 2016. The effect of intermittent renewables on the electricity price variance. OR Spectrum 38(3): 687-709. ISSN 14366304.

Zhou, Y., A. Scheller-Wolf, N. Secomandi, S. Smith. 2016. Electricity trading and negative prices: Storage vs disposal. Management Sci. 62(3): 880-898. ISSN 0025-1909. 